\title{
GENEROSOS Y EMPÁTICOS O ¿PRAGMÁTICOS Y UTILITARISTAS?: LOS ARGUMENTOS DEL ADULTO MAYOR CHILENO FRENTE A UN DILEMA SOCIAL*
}

\author{
Cristián Santibáñez \\ Universidad Católica de la Santísima de Concepción \\ csantibanez@ucsc.cl
}

Recibido: 15/05/2018 - Aceptado: 18/10/2018

doi.org/10.17533/udea.lyl.n75a09

\begin{abstract}
Resumen: En este trabajo se discuten algunos resultados de una investigación sobre la facultad argumentativa en adultos mayores (sobre 65 años de edad) de tres regiones de Chile, particularmente las respuestas escritas a un dilema social. Los resultados indican que aquellos adultos mayores que optan por empatizar con el protagonista del dilema social son en su mayoría mujeres, mayores de 70 años de edad y con un nivel educativo más alto. Sus argumentos muestran mayor densidad en términos de la cantidad y pertinencia de razones para apoyar el punto de vista.
\end{abstract}

Palabras clave: adulto mayor; argumento; lexicometría; palabras clave.

\section{GENEROUS AND EMPATHIC, OR PRAGMATIC AND UTILITARIAN? THE ARGUMENTS OF CHILEAN ELDERLY PEOPLE CONFRONTED WITH A SOCIAL DILEMMA}

\begin{abstract}
This paper discusses some results of a research on the argumentative faculty in older adults (aged over 65) from three regions of Chile, particularly their written responses to a social dilemma. The results indicate that older adults who choose to empathize with the protagonist of the social dilemma are mostly 70-year-old women and more educated. Their arguments show greater density in terms of the quantity and relevance of reasons to support their point of view.
\end{abstract}

Keywords: Elderly People; Argument; Lexicometry; Keywords.

\footnotetext{
* Artículo producido en el marco del Proyecto Fondecyt Regular 1170492, Caracterización lingüística y cognitiva de la competencia argumentativa en adultos mayores: un estudio en las regiones del Bio-Bio, Coquimbo y Metropolitana.
} 


\section{Introducción}

$\mathrm{E}$ ste trabajo reporta algunos de los resultados de una investigación de tres años que busca caracterizar, tanto desde el punto de vista cognitivo como lingüístico, la competencia argumentativa del adulto mayor en Chile. La investigación se concentra en tres regiones del país: Bio-Bio, Metropolitana, y Coquimbo, las dos primeras las más populosas e importantes desde el punto de vista social y económico.

Como muestran los datos del Instituto Nacional de Estadísticas en Chile (INE, 2017), desde mediados de la década de 1990 a la actualidad, se observa una gran transformación de la población en el país, que requiere un esfuerzo científico multidimensional para responder adecuadamente al desafío que significa tal cambio en la estructura demográfica. Los datos que en la sección 2 del trabajo se reportan, obligan a pensar cómo preparar las condiciones económicas, sociales y culturales para interactuar con este creciente grupo social. Entre las necesidades más significativas de los adultos mayores (en adelante AM), destaca con singular importancia la existencia de servicios sociales y de salud, pues la vejez se caracteriza por la aparición de un alto número de patologías crónicas que limitan la autonomía y que requieren de un mayor número de atenciones médico-sociales.

El número de personas que ha superado la edad mínima de jubilación y que aun así participa del mercado laboral en calidad de ocupados, ha experimentado un fuerte aumento en Chile. Frente a este escenario, surge la pregunta de cómo puede aportar la ciencia del lenguaje en la creación de mecanismos y políticas que retroalimenten y faciliten la interacción pertinente con el adulto mayor. Si bien la respuesta compromete varias dimensiones importantes, una de ellas emerge como fundamental: la competencia argumentativa. Lograr una descripción y explicación adecuada y sistemática de la competencia y conducta lingüística de argumentar de los AM contribuirá decididamente en aquella tarea, ya que el adulto mayor seguirá tomando decisiones fundamentales, participando de discusiones de carácter social que exigen mantener cierto nivel de capacidad argumentativa, interactuando con generaciones más jóvenes que toman como orientación varias de sus visiones. Desde el punto de vista de la disciplina lingüística, contar con una caracterización de la competencia argumentativa de este grupo etario permitirá ir avanzando en una mirada ontogénica y dinámica tanto de esta facultad como del funcionamiento del lenguaje en general.

Bajo el prisma que da el párrafo anterior, los objetivos específicos de este trabajo son: 1) Analizar y describir el uso argumentativo de tópicos recurrentes frente a la respuesta de un dilema social; 2) Describir las tendencias de los tipos argumentos 
según variables socio-económicas y educativas; y 3) Describir el uso de estructuras adversativas en los argumentos utilizados en los puntos de vista de los AM al responder el dilema social.

Para alcanzar estos objetivos, el trabajo se organiza de la siguiente forma: en la sección 2 se ofrece una serie de datos estadísticos que justifican la selección de la unidad de observación; en la 3, se discute algunos alcances teóricos y hallazgos empíricos — cognitivos y lingüísticos - relativos a distintas ecologías argumentativas etarias que pueden orientar la lectura de los datos tanto para ver ciertas continuidades como diferencias; en la 4, se detalla el encuadre metodológico que permitió obtener los resultados que se exponen luego; en la sección 5, se exponen los resultados utilizando un análisis léxico-métrico, detallando tendencias generales, y luego analizando en términos argumentativos y de palabras clave en los argumentos los distintos grupos de AM que se obtienen según la respuesta al dilema social; y en la 6, se discute los principales hallazgos y se concluye observando qué elementos específicos debieran seguir profundizándose para acercarnos a una descripción más robusta del modo en que se expresa la facultad argumentativa en personas mayores de 65 años. Para apoyar la discusión en el apartado 6, se utilizan algunos conceptos que forman parte de la literatura relativa al estudio de dilemas sociales y morales, particularmente en lo relacionado con castigos, recompensas y otras estrategias de sanción colectiva.

\section{Antecedentes generales sobre envejecimiento en Chile}

Chile envejece de forma sistemática y muy rápidamente. De acuerdo con el reciente Censo poblacional de 2017, la relación entre mayores de 65 años y menores de 14 se ha triplicado entre 1992 y 2017 . Hoy de cada 10 menores de 15 años, hay 6 adultos mayores de 64 años. Las proyecciones de 2015 del Instituto Nacional de Estadísticas (INE) chileno ya habían señalado que el país tendrá hacia el 2020 la esperanza de vida más alta del mundo junto a Estados Unidos. De acuerdo con tales proyecciones, la edad promedio de esperanza de vida sería de 82,2 años para las mujeres y de 77,4 para los hombres. Según el Enfoque Demográfico de Género del INE, hacia el 2020 Coquimbo (una de las regiones que se reporta en este estudio, junto con las del Bio-Bio y Metropolitana), será el área del país que más concentrará población con mayor proyección de vida, tanto de hombres (78,18 años) como de mujeres (82,97 años).

En relación al índice de envejecimiento demográfico medido por el índice de adulto mayor (IAM), en el año 2020 será mayor para las mujeres en Chile. El INE concluye que el incremento de la población femenina mayor de 60 años implica un 
envejecimiento demográfico que exige medidas que deben ser abordadas a través de políticas públicas para satisfacer sus necesidades. Los datos demuestran que en el año 2007 en el país había 48 AM por cada cien menores de 15 años, es decir, un adulto mayor por cada 2 menores de 15 años. Las estimaciones indican que hacia el 2025 en Chile habrá un adulto mayor por cada menor de 15 años y, hacia el 2050, dicha cifra prácticamente se duplicará por cada menor de 15 . El envejecimiento de la población chilena presenta como característica adicional un mayor aumento de la población de 80 años y más.

\section{Adulto mayor: teoría de la argumentación, lenguaje y cognición}

El mainstream de la teoría de la argumentación no ha producido conocimiento sobre lo que ocurre con la competencia argumentativa del adulto mayor. Sí la hay respecto de otros grupos etarios: niños pequeños, jóvenes universitarios y adultos (van Eemeren, Garssen \& Meuffels, 2009; Hample \& Anagondahalli, 2015; Mercier, 2016; Migdalek, Santibáñez \& Rosemberg, 2014; Santibáñez, 2014; Shär, 2011, 2017). Los estudios de diferentes comunidades de hablantes según un criterio de edad - criterio que es una de las formas para situar el problema del desarrollo y manifestación de facultades cognitivas_- debieran ser capaces de despejar: 1) qué grado de complejidad tiene la práctica argumentativa del adulto mayor (por ejemplo, cuántas razones se esgrimen en un punto de vista, qué tipos de esquemas argumentativos son utilizados, cuán relevantes o suficientes son las razones para apoyar el punto de vista ); 2) qué función le da a esta posibilidad lingüística el adulto mayor y qué actitudes comunicativas, teóricamente entendidas como marcos argumentativos, están asociadas en el momento en que manifiesta un acto de habla argumentativo (cierta tendencia a la cooperación, o a la agresividad verbal, por ejemplo).

Respecto de complejidad argumentativa, y considerada desde una perspectiva dinámica, cabe entenderla normativamente como la densidad o cantidad de entramados justificativos en la producción argumentativa (arbóreamente: proposición/ punto de vista, soporte/razón, explicación y justificación; Hammer \& Noemi, 2015). Contextualmente, la complejidad argumentativa se podría definir como la capacidad del hablante de producir argumentos suficientes, relevantes y aceptables de acuerdo con el problema bajo discusión, la situación y la audiencia a la que va dirigida la argumentación (Johnson, 2000; Tindale, 2004).

Respecto de la función de argumentos y argumentación, la teoría argumentativa ha avanzado sistemáticamente. Por ejemplo, la teoría de los bloques semánticos (Ducrot, 2004; Ducrot \& Carel, 2006; García Negroni, por aparecer), considera la argumentación como un proceso polifónico de enunciación, en cuyo seno se juegan las 
marcas lingüísticas y semánticas de los tópicos que una sociedad o grupo comparte. Esta perspectiva será de ayuda más adelante cuando se analicen los datos obtenidos.

Algo similar ocurre, desde un punto de vista funcional, con el enfoque pragmadialéctico de la argumentación (van Eemeren \& Grootendorst, 2004; van Eemeren, 2010), donde la argumentación es definida como una forma para resolver críticamente una diferencia de opinión, es decir, es una teoría en la que la funcionalidad de la argumentación es aún más específica respecto de la teoría de los bloques semánticos, pues se trata incluso de declarar, por ciertos procedimientos -0 reglas para la discusión crítica - a una de las partes como depositaria de la razón respecto de la discusión en cuestión. Para la Escuela de Windsor (Johnson, 2000; Johnson \& Blair, 1977; Walton, 2007, 2013) la lógica informal se ha entendido como un brazo de la lógica cuya función, tarea y objetivo es desarrollar estándares no-formales, criterios y procedimientos para el análisis, interpretación, evaluación, crítica y construcción de la argumentación en el discurso cotidiano.

Desde la psicología cognitiva ha habido perspectivas interesantes respecto de la función atribuida a la argumentación. Particularmente (Sperber \& Mercier, 2012; Mercier, 2011a, 2011b; Mercier \& Sperber, 2017), se ha puesto en duda algunos avances provenientes de la teoría de la argumentación entre lingüistas y filósofos, al sostener que la argumentación y la inteligencia corren por módulos paralelos y no siempre funcionan de forma coordinada, por lo que los resultados de un proceso argumentativo no están determinados por una evaluación estrictamente crítica; del mismo modo, sostienen que los humanos argumentamos siempre con un sesgo de confirmación y que la evidencia en psicología cognitiva de los últimos cuarenta años muestra que, debido a este sesgo, los humanos argumentamos aquello que podemos justificar y no lo que sabemos sería una mejor opinión o argumento. Así concebida la actividad por Sperber y Mercier, apoyada por una vasta gama de experimentos y de datos citados por los autores, la argumentación no brinda ninguno de los beneficios y funciones positivas que a menudo se piensa tiene. Una de las conclusiones que más llama la atención de la posición de Sperber y su equipo, es que los humanos son en principio crédulos de aquellos en los que confían, pero tan luego como desconfían lo suficiente, recién allí resuelven cotejar más atentamente el contenido de los argumentos, poniendo en práctica una revisión de la coherencia y relevancia de lo comunicado. Vigilancia epistémica es el término escogido para la última acción descrita (Sperber et al., 2010).

En virtud de esta discusión teórica sobre complejidad y funcionalidad argumentativa (Santibáñez, 2015), que aún sigue desarrollándose (Hu, 2017), la propuesta en este trabajo es que funcionalmente la argumentación cabría entenderla como una 
actividad colectiva que, principal pero no exclusivamente, se manifiesta verbalmente (van Eemeren \& Grootendorst, 2004), y cuyo objetivo se plantea como la resolución de diferencias de opinión a partir del intercambio crítico de puntos de vista entre sujetos que admiten tales diferencias. La capacidad de argumentar la utiliza un individuo cuando busca, tácita o manifiestamente, hacer que su oyente, entre otras posibilidades, cambie de opinión o tome determinado curso de acción. Que la competencia argumentativa se exprese como una actividad eminentemente colectiva, significa que en ella opera como requerimiento estructural, lo que se conoce como intencionalidad colectiva (Tomasello, 2014), vale decir, aquella intencionalidad que crea interacciones humanas a partir de una coordinación, automática o reflexiva, entre participantes que tienen metas comunes. En el caso de la argumentación, lo que se intenta resolver colectivamente es una controversia, incluso cuando algunos de los participantes estén motivados por la diversión de contraargumentar, u otra meta de carácter individualista.

Desde una perspectiva más empírica, que es de mayor relevancia para el estudio que aquí se reporta, la noción de marco argumentativo (Hample, 2005) es de gran utilidad, tanto del punto de vista funcional como del interactivo y/o el dinámico. Este concepto pone en juego elementos comunicativos pero también cognitivos, y su rendimiento tanto conceptual como empírico está asociado al análisis de las actitudes que los sujetos tienen sobre esta práctica; este constructo se apoya, además, en una idea de argumentatividad, esto es, en una medida actitudinal acerca de la aproximación-evasión hacia la argumentación. Por ejemplo, y como la bibliografía existente ya demuestra, las personas con alto grado de argumentatividad están más dispuestas a participar en intercambios argumentativos, por lo que buscan oportunidades para defender sus argumentos o desafiar los argumentos, razonamientos o evidencias de los otros (Xie, Hample \& Wang, 2015). Un marco argumentativo es un patrón comunicativo que manifiesta la actitud subjetiva que tiene un individuo sobre la práctica argumentativa, y que deviene en una esquematización socio-mental que procesa los mensajes de los demás, vale decir, funciona como un tipo de herramienta cognitiva.

Se debe tener presente que en virtud de diferentes coordenadas socio-culturales y demográficas, las distintas comunidades de habla harán un uso distinto de la facultad porque los desafíos son disímiles (lo que la literatura al respecto ha demostrado en otras realidades, véase Hample, 2005; Hample \& Anagondahalli, 2015; Hample, Warner \& Young, 2009; Ricco \& Sierra, 2011; Santibáñez \& Hample, 2015), de modo que es esperable que el adulto mayor en Chile argumente de forma distinta respecto de otros grupos etarios, intuición que esta investigación busca justamente esclarecer (corroborar o falsificar) empíricamente. 
En relación con personas adultas (ya que respecto de infantes y adolescentes se puede consultar Peronard, 1991; Crespo, 1995; Migdalek, Santibáñez \& Rosemberg, 2014; Parodi, 2000), y considerando particularmente estudiantes de enseñanza superior, varios estudios han aportado en la caracterización de la facultad argumentativa. Se ha observado (Santibáñez \& Hample, 2015), que los estudiantes universitarios chilenos se caracterizan por no adherir a la actividad argumentativa un valor de corrección de creencias y resolución de conflictos. Del mismo modo (Santibáñez, Rosemberg \& Migdalek, 2016), identificaron que a partir del tercer año universitario, las personas comienzan a mostrar una creciente densidad argumentativa vía la articulación de puntos de vista, el uso de conectores causales y de conectores condicionales. Como sostuvieron los autores citados, esta densidad argumentativa que se manifiesta a partir del tercer año de vida universitaria, probablemente se relaciona tanto con un tipo de madurez cognitiva como con una actitud de compromiso más asumido del agente que tendría mayor conciencia de sus actuaciones argumentativas públicas y con una práctica argumentativa más exigente (tomar posición, defender ideas propias, poner a prueba una capacidad inventiva y creativa). Estas variables (madurez cognitiva — por ejemplo, vinculada con sofisticación sintáctica y léxica—, predisposición, desarrollo escolar, y exigencia de contexto) deben ser investigadas con precisión.

Parte de la precisión que debe resguardarse, se obtiene con la inclusión de más variables lingüísticas, sensiblemente pragmáticas, para efectos de la construcción de los instrumentos de recolección de información, como para el análisis, particularmente cuando la competencia argumentativa se estudia en sujetos AM. Esto es así ya que la literatura en el ámbito señala que el adulto mayor presenta particularidades que hacen prudente establecer las relaciones posibles entre, por ejemplo, complejidad argumentativa y la duración de las pausas o los tiempos de latencia en contextos de controversia. Respecto de esto último, se sabe que en el desempeño fonéticofonológico los AM con indemnidad cognitiva no parecen presentar dificultades. Básicamente, los AM no presentan diferencias en la producción de los sonidos del habla comparados con sujetos con enfermedad de Alzheimer o con adultos jóvenes. Sin embargo, se sabe también que ocurren cambios en el rendimiento de otras habilidades lingüísticas.

En relación con el léxico, diferentes estudios indican que se observa una conservación del léxico pasivo en los AM (Wingfiel \& Stine-Morrow, 2000) y un mejor rendimiento que los jóvenes en pruebas estándares de vocabulario (Kemper y Sumner, 2001; Singer, Verhaeghen, Ghisletta, Lindenberger \& Baltes, 2003). No obstante, las personas mayores pueden experimentar dificultades de acceso léxico para hallar nombres propios (Condret-Santi et al., 2015) o en tareas de búsqueda de palabras, lo que se ha denominado fenómeno de punta de la lengua. Diversos 
estudios muestran que este fenómeno es particularmente sensible a los efectos de la edad (Gollan \& Brown, 2006; Juncos et al., 2006), lo que se explicaría por déficits que sufren los mayores en la recuperación de las representaciones fonológicas y no por una disminución de su caudal léxico, que más bien tiende a aumentar (Burke \& Shafto, 2008; Martín-Aragoneses \& Fernández-Blázquez, 2012).

A nivel sintáctico, las investigaciones actuales muestran que el procesamiento sintáctico inmediato parece no afectarse con el envejecimiento, pero disminuye la efectividad de los procesos postinterpretativos o fuera de curso (Dede, Caplan, Kemtes \& Waters, 2004; Caplan, Dede, Waters, Michaud \& Tripodis, 2011), lo que se refleja en más dificultades para recuperar y usar la información sintáctica en otras tareas (Véliz, 2004; Riffo \& Benoit, 2007; Véliz, Riffo \& Vásquez, 2009; Véliz, Riffo, Aguilar \& Sáez, 2011; Arancibia, Véliz, Riffo \& Roa-Ureta, 2014). En producción sintáctica, el panorama es distinto, pues — al menos en inglés - hay abundante evidencia (aportada tanto por estudios descriptivos como experimentales) de que con la edad disminuye la longitud y la complejidad sintáctica de las oraciones, tanto en el discurso escrito como oral (Kemper, 1992; Kemper, Thompson \& Marquis, 2001; Kemper, Herman \& Lian, 2003; Kemper, Herman \& Chiung-Ju, 2004). En Chile Véliz, Riffo, Hernández, Sáez y Sáez, (2013) aportan los mismos resultados en un estudio experimental de producción de oraciones de sintaxis compleja.

Cuando se ha analizado el discurso oral y escrito de los AM y de grupos de jóvenes, se ha observado que los primeros presentan dificultades en la producción de ciertos tipos de discurso. La densidad de las ideas disminuye en ensayos autobiográficos escritos (Kemper et al., 2001) y en descripciones orales de imágenes (Mackenzie, 2000). En el ámbito hispánico, Juncos et al. (2005) encontraron que con la edad se reduce la densidad del contenido informacional y las referencias cohesivas de las narraciones y aumentan los contenidos irrelevantes. Por su parte, Ulatowska et al. (1985) señalaron que los AM presentan problemas cuando deben volver a contar historias, resumir textos o establecer la moraleja de una historia. En Chile, Tapia-Ladino (2005) señala que los AM con indemnidad cognitiva producen mayor cantidad de palabras que los adultos jóvenes frente a estímulos como una pregunta y la descripción de una imagen. Sin embargo, los jóvenes incorporan más información nueva, mientras que los AM tienden al circunloquio o la repetición de palabras. Sobre los estudios de comprensión del discurso, se ha señalado que los AM presentan un menor rendimiento en tareas de recuperación de volúmenes de información y en pruebas de reconocimiento de textos leídos o escuchados. Por su parte, Mackenzie (2000) reportó que la precisión en respuesta a preguntas sobre detalles, inferencias o metáforas presenta diferencia con los jóvenes, pero las diferencias aumentan cuando se compara con AM de más de 75 años en quienes se advierte una disminución en la capacidad de procesamiento semántico. 
A partir de esta información, se pueden establecer algunas constantes en los hallazgos señalados. En los AM con indemnidad cognitiva se observa una preservación del conocimiento fonológico y léxico-semántico; dificultades en el acceso léxico y recuperación fonológica; un declive de la producción sintáctica y dificultades para recuperar el significado de oraciones una vez procesadas. En relación con los métodos más utilizados para evaluar el uso del lenguaje en los AM se destaca el empleo de pruebas estructuradas, tales como la confrontación de nombres (Wilson et al., 2002), la producción de palabras aisladas (Fabriogoule et al., 2006), o la generación de palabras que comienzan con una letra determinada (Palmer et al, 2006). Igualmente, destaca la recurrencia de estudios experimentales de diversa índole, particularmente en los estudios sobre los aspectos sintácticos (Arancibia et al., 2014; Dede et al., 2004).

Uno de los aspectos cruciales que permite apreciar toda la discusión teórica y bibliográfica precedente, en perspectiva ontogénica, es que para caracterizar de mejor manera la competencia argumentativa debemos construir nuestros métodos con una orientación interactiva y social, y procurar que fenómenos pragmáticos, semánticos y léxicos sean parte de los recursos a los que el hablante debe enfrentarse cuando participa en una tarea cuasi o experimental de una investigación. Es en este sentido que la tarea que a continuación se describe, y de la que se reportan los análisis, fue concebida. En relación con los aspectos conceptuales sustanciales (función de conflicto cognitivo, tipo de esquemas, estrategias utilizadas, etc.) las secciones $5 \mathrm{y}$ 6 darán cuenta.

\section{Metodología}

\subsection{Descripción general}

La investigación se planteó como un estudio de tipo descriptivo-explicativo, basada en una combinación metodológica mixta cuantitativo-cualitativo, y de la cual aquí se reporta el análisis de la base de datos cuantitativos, en particular la encuesta, en la que se incluyó el ítem dilema social que más adelante se detalla.

\subsection{Participantes}

La muestra es de carácter no probabilística. Estuvo compuesta por AM de las regiones de Bío-Bío, Coquimbo y Metropolitana. Se accedió a AM según registros de establecimientos de salud, el Servicio Nacional del Adulto Mayor del Gobierno de Chile (SENAMA), Colegio de Profesores del Bío-Bío, Intendencia de Coquimbo 
y a partir del método de bola de nieve. Los participantes debían cumplir con los siguientes criterios de inclusión no probabilística:

1. Edad: AM mayor de 65.

2. Nivel socioeconómico: pertenencia a los 4 grupos socio-económicos superiores, exceptuando así participación de población de extrema vulnerabilidad que se encuentra en clara desventaja para responder sistemáticamente los instrumentos de la investigación y para resguardar la seguridad del trabajo de campo.

3. Desempeño cognitivo: AM con indemnidad cognitiva, es decir, sin indicios de demencia senil o deterioro cognitivo asociado a patologías (vigentes o pasadas).

4. Consideraciones éticas: firma de consentimiento informado.

\subsection{De los instrumentos de selección de muestra}

\subsubsection{Instrumentos para la selección de la muestra}

Para asegurar que los participantes cumplieran con el criterio de indemnidad cognitiva, se utilizó como medios de tamizaje/selección los siguientes instrumentos, ambos de administración individual:

1. Escala Abreviada de Depresión Geriátrica Yesavage: en su versión abreviada de 5 ítems (Hoyl et al., 2000), que ha sido probada en población chilena con una sensibilidad de 0,88 y una especificidad de 0,90 . Su eficiencia diagnóstica (o proporción de resultados correctos) es de 0,89 . Se descarta al participante si su puntaje es inferior a 3 .

2. Test Minimental (Quiroga, Albala \& Klaassen, 2004), versión abreviada: evalúa diversas funciones que dan cuenta de la normalidad cognitiva del individuo: orientación temporal y espacial, memoria de corto y largo plazo, capacidad ejecutiva, atención, abstracción, comprensión, memoria e inteligencia y capacidad viso-constructiva. El puntaje total de la aplicación es de 19 puntos. Se considera alterado si el puntaje es menor de 13 puntos.

\subsubsection{Del instrumento cuantitativo para el desarrollo del estudio}

Una vez que se seleccionó la muestra de participantes, se les aplicó el cuestionario construido con preguntas cerradas codificadas en combinación con método 
de escalamiento Likert y diferencial semántico para medir actitudes y función atribuida al argumento y a la argumentación. El instrumento midió variables específicas, entre otras: Identificación socio-demográfica básica: edad, género, ingreso familiar, años de escolaridad, biografía laboral y de participación pública (de la comuna, instituciones deportivas, por ejemplo); Argumentatividad: disposición a participar en la actividad de argumentar; Agresividad verbal: uso de estrategias de ataque para enfrentar situaciones de controversia; Marcos argumentativos: identificación de meta-objetivos que los sujetos reconocen como razones para argumentar (Hample, 2005); Individualismo/colectivismo: identificación de valores culturales asociados a la práctica argumentativa; Poder: identificación de función del argumento y la argumentación en situaciones jerárquicas; Auto-constricciones: reconocimiento de limitaciones y/o motivaciones sociales y cognitivas para argumentar; Cortesía: medición de tendencias de cuidado a terceros en situaciones de discusión. Para disponer de información escrita y con el objetivo de poner en una perspectiva más interactiva y social el auto-reporte de los encuestados, se incluyó un dilema social que debían responder. El dilema social fue el siguiente: "Por favor, lea el siguiente párrafo, y escriba su opinión en relación con la consulta que se hace: "Un hombre había sido condenado a 10 años de prisión. Después de un año escapó del centro penitenciario cambiándose el nombre por López. Durante ocho años trabajó duramente y, poco a poco, pudo ahorrar el dinero suficiente para montar su propio negocio. Era honesto con sus clientes. Pagaba altos salarios a sus trabajadores y daba la mayor parte de sus beneficios para obras de caridad. Pero un día el señor González, un antiguo vecino de López, le reconoció como el hombre que había escapado de la prisión ocho años antes y al que la policía estaba buscando. ¿Debería el señor González denunciar al señor López a la policía e ir éste de nuevo a prisión?” ¿Cree usted que debe el señor González denunciar al señor López? Fundamente su opinión".

De las 244 encuestas realizadas en las tres regiones de Chile, 228 participantes respondieron el dilema social. 16 participantes no respondieron, particularmente por cansancio o falta de tiempo. Las 228 respuestas al dilema contenido en la encuesta fueron analizadas con un software léxico-métrico, que a continuación se describe.

\subsection{Herramienta de análisis: Software TLAB Plus Léxico-métrico}

El software TLAB Plus está especialmente diseñado para rescatar los aspectos comprensivos de los discursos y textos. El software permite rescatar los tipos de palabras utilizadas paradigmáticamente en términos de promedio, y la serie de 
conjugaciones efectuadas en las que ellas aparecen. Como se sabe, la lexicometría es un análisis cuantitativo de última generación, que utiliza métodos estadísticos para el tratamiento de datos textuales a través de diversos algoritmos, muchos de ellos sobre la base de desarrollos en inteligencia artificial. La lexicometría se puede clasificar en: 1) métodos de descripción (qué cosas se dicen, quién lo dice, cómo lo dice); 2) métodos de asociación (qué ideas/conceptos/opiniones están relacionadas); y 3) métodos de segmentación (cómo se agrupan las ideas/conceptos/opiniones en grupos estadísticamente significativos). La lexicometría se alimenta de textos, y éstos pueden provenir de las más diversas fuentes: transcripciones de entrevistas, grupos focales, respuesta a preguntas abiertas, comentarios en redes sociales, etc. Los análisis que a continuación se presentan respecto de las respuestas de los AM al dilema social, precisamente dan cuenta de los tres niveles de clasificación en lexicometría: descripción, asociación, y segmentación. Se ha hecho, además, cruzando tales análisis con variables nominales que la encuesta contenía en su dimensión socio-económica.

\section{Principales hallazgos}

\subsection{Respuestas dominantes}

A partir de los datos que entrega tanto el análisis léxico-métrico y la estadística de las variables socio-económicas se obtienen datos importantes respecto de las posiciones asumidas. Los datos relativos a la distribución básica de las respuestas, arroja los siguientes grupos y sus porcentajes: un $68 \%$ de los AM rechaza que González denuncie a López, un 23\% de los AM aprueba la denuncia, y un 9\% no tienen claro si rechazar la denuncia o denunciar. En un análisis más detallado de esta distribución que se obtiene sólo en términos de frecuencias y porcentajes de tales frecuencias según género, edad, educación y región de Chile (vale decir, sin análisis de correlaciones para corroborar o falsificar cómo una variable influencia otra), hay cierta información que es relevante como telón de fondo del análisis que se muestra posteriormente. Por ejemplo, que en el grupo de quienes rechazan que González denuncie a López, destaca que el porcentaje más alto son mujeres, que en su mayoría están por encima de los 70 años, relativamente más educadas y de la capital del país (ciudad de Santiago, región Metropolitana). Por su parte, quienes optan por que González denuncie a López, son mayormente hombres, menores de 70 años, con menor educación y de las regiones del Bío-Bío (ciudad de Concepción) y de Coquimbo (ciudad de La Serena). Entre quienes no saben qué posición tomar, emerge que son en su mayoría mujeres, con más de 70 años, claramente con un nivel más alto de educación y de la capital del país. 


\subsection{Argumentos centrales y asociaciones léxicas}

En relación con los argumentos que se encuentran en los tres grupos (los que apoyarían la denuncia de González, los que no saben si hacerlo y los que rechazan que González denuncie a López), el análisis léxico-métrico permite recuperar los nodos argumentativos centrales en distintos niveles de expresión. Cabe señalar que los análisis del tercer grupo (los que rechazan que González denuncie), serán más detallados y, en cierta medida, más abundantes, debido a que por el tamaño se pueden realizar más acercamientos estadísticos y agrupaciones (que en terminología léxicométrica se conocen con la noción de cluster, esto es, agrupamiento). Se comenzará con el análisis de los AM que argumentan a favor de que González denuncie a López, discutiendo en primer lugar el análisis de escalado multidimensional y, en segundo lugar, el análisis semántico de asociación estadística. Luego, se profundiza en el grupo de AM indecisos en sus argumentos respecto de si apoyarían a González en su denuncia. Finalmente, se procedió al análisis de los AM que rechaza que González denuncie. Cabe recordar que el escalado multidimensional permite entender cómo se agrupan las palabras utilizadas, resumiendo de manera consistente y paradigmáticamente muy fiel la manera en que se distribuirían las categorías gramaticales más importantes de, en este caso, los argumentos de los AM. En el despliegue visual de los escalados multidimensionales, las medidas de stress representan la calidad del resultado obtenido y, por lo tanto, la fuerza en que se producen las agrupaciones entre los diversos lemas o codificaciones de palabras claves. Mientras el índice esté más cerca de cero, mejor será la calidad y precisión de los resultados.

\subsubsection{AM que apoyan con sus argumentos que González denuncie a López}

Los argumentos utilizados por este grupo se pueden desplegar visualmente utilizando la siguiente disposición:

Los ejes $(x$ e $y$ ), como se sabe respecto del análisis de escalado léxico-métrico, no representan variables, solo permiten desplegar las agrupaciones de palabras más representativas en cuadrantes de forma tal que sea visualmente posible capturar el despliegue semántico. Si se tuviera que reconstruir de manera sucinta los puntos de vista paradigmáticos en relación con la proximidad semántica de ocurrencia de palabras clave, y como se destaca en los sub-cuadros (rojo, verde, azul y gris), se obtendría enunciados como: Creo que es correcto cumplir la condena; la persona debe cumplir con la ley; la persona cometió un delito; debe pagar la deuda con la justicia. Ahora bien, y desde un punto de vista técnico (Walton, 2013) de reconstrucción de argumentos, la configuración podría ser la siguiente, en tanto tipo de argumento serial: 
Escalado multidimensional 1

Sí apoyan que González denuncie a López
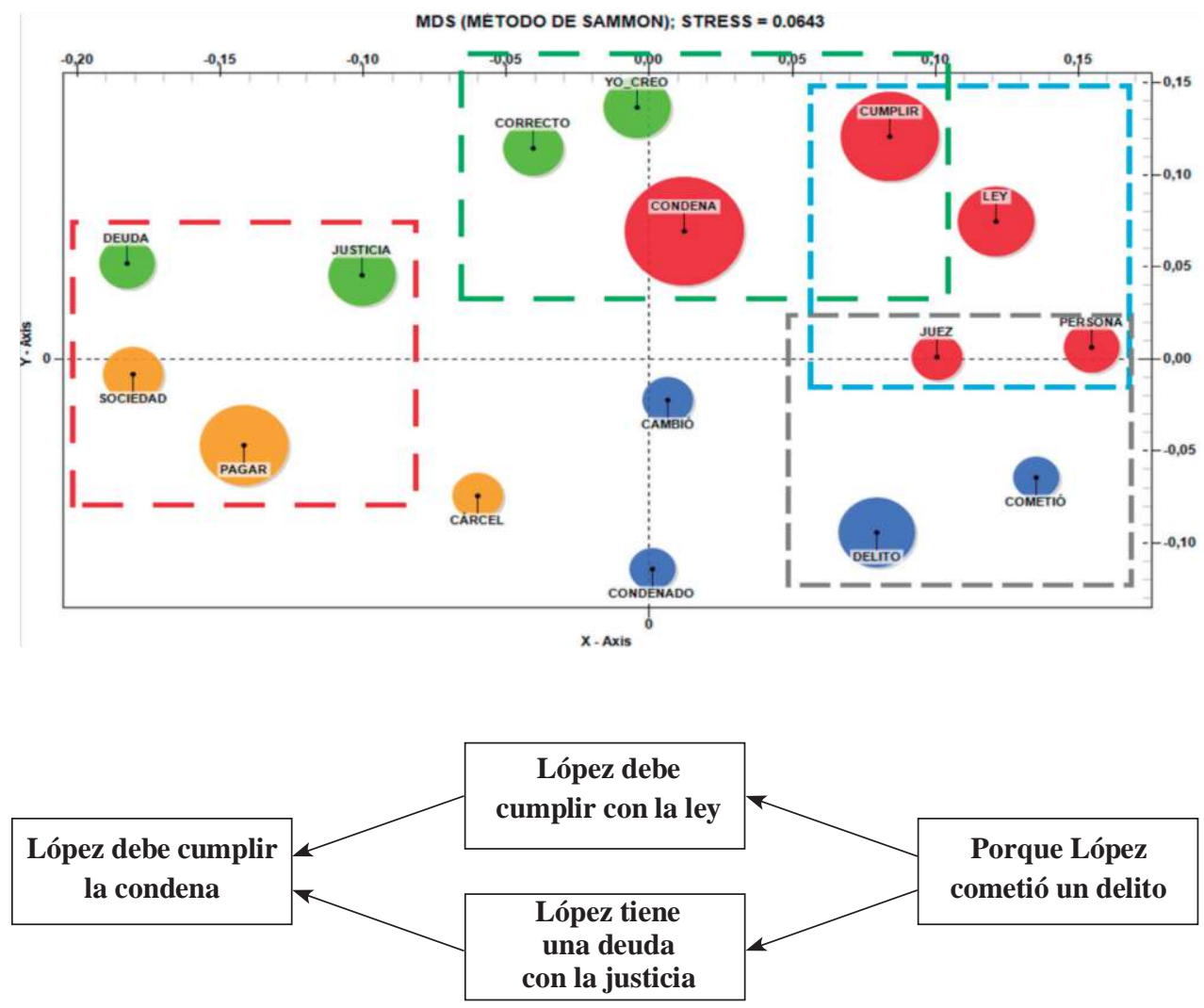

Figura 1. Argumento Serial

Por qué apoyar que González denuncie a López

Los AM utilizan el dato de la responsabilidad de un delito para sostener dos afirmaciones que apoyan el punto de vista final que también (como el dato inicial: que $x$ hizo $y$ ) tiene un perfil de consecuencia práctica. En un argumento serial, un primer elemento del entramado argumentativo justifica la exposición de un punto intermedio, que a su vez respalda la conclusión, como se observa en la figura 1. Ahora bien, desde la perspectiva de la lógica informal para evaluar argumentos, se podría calificar que el dato siendo muy relevante y aceptable, no es suficiente para sostener con fuerza el punto de vista sostenido. De aquí que, como se verá luego, los otros dos grupos fortalezcan sus posiciones utilizando razonamientos (Toulmin, Rieke \& Janik, 1979) que conjugan este dato con otros, o simplemente lo relativizan. 
Respecto del análisis semántico de asociaciones específicas de este grupo, veremos a continuación las palabras clave y su red de vínculos, al mismo tiempo que se dan citas (recuadro al frente, en lo sucesivo con los otros grupos se sigue de la misma manera) de los dichos por lo AM que grafican lo que la asociación semántica muestra:

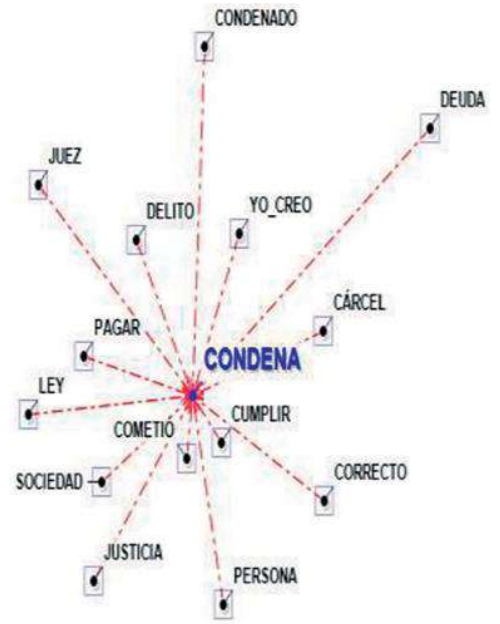

Subjact: LFMMA ASSOCLATIONS < COMEHO > AND $<$ CONDENA $>A N D<C$ CUIPLIR >

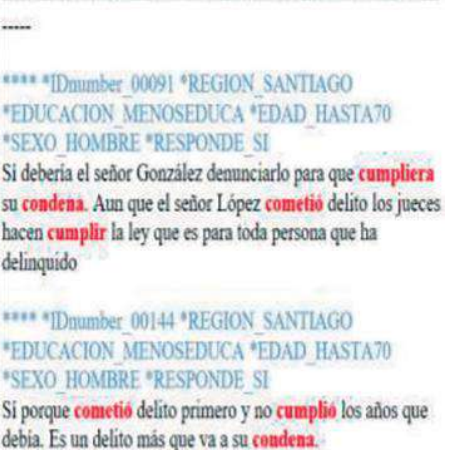
debia. Es un delito más que va a su coudena.

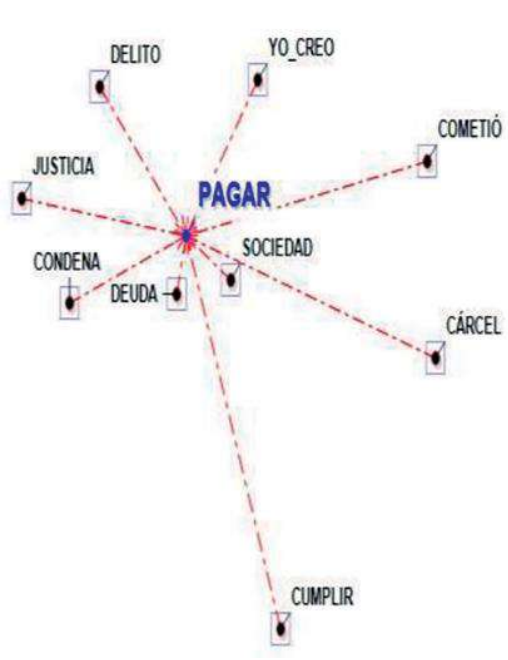

Subject: LFMMA ASSOCIATIONS $<$ DEUDA $>$ AND $<$ PAGAR > AND < SOCIEDAD >

*IDnumber 00054 *REGION SANTIAGO

"EDUCACION MENOSEDUCA "EDAD HASTA70 "SEXO_HOMBRE "RESPONDE SI

Dada a la experiencia del señor López conocen el lado obscuro de la luna ( cárcel) hace cambios en el ser humano Tiene una deuda con la sociedad que debe pagar. No estar impune con su ser interior y realmente será libre

w** *IDnumber 00074 *REGION SANTIAGO "EDUCACION MENOSEDUCA *EDAD HASTA70 "SEXO HOMBRE 'RESPONDE SI

Si, el señor Gonzalez debe denunciar al señor López para que pague su deuda con la sociedad, en ese momento la justicia debe analizar las buenas obras que realiza el señor López y podria dar una nueva condena o perdonarlo.

*** *IDnumber 00134 *RFGION SANTIAGO *EDUCACION MENOSEDUCA *EDAD HASTA70 "SEXO HOMBRE 'RESPONDE SI

Deberia, pero señor López se rehabilitó, pero debe pagar su deuda a la sociedad 


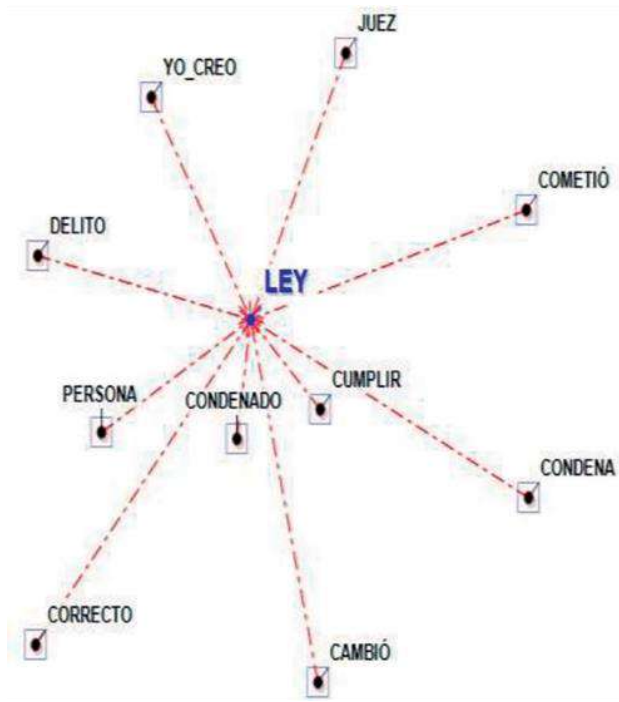

Subject : LEMMA ASSOCIATIONS $<$ CUMPLIR $>$ AND $<$ LEY $>$ AND $<$ CONDENADO $>$

***** *Daumber_00176 *REGION SANTIAGO

*EDUCACION_MENOSEDUCA *EDAD_HASTA70

*SEXO_HOMBRE *RESPONDE_SI

Legal y moralmente debe hacerlo, pero con el agravante de demostrar su cambio total a lo que fue antes, para que asi la ley lo revaluare y posiblemente no lo sometería a prisión. Legalmente porque no habia terminado su estadia impuesta por la ley pues escapo. Moralmente porque la ley debe respetarse y hacerse cumplir, pues, aunque cambió le debia a ella, la penalidad que se le decreto por los motivos por el cual fue condenado.

Figura 2. Asociaciones semánticas específicas y dichos ejemplares de AM que apoyan que González denuncie

Las asociaciones corroboran lo que el escalado multidimensional muestra, al mismo tiempo que los ejemplos concretos de dichos de AM de este grupo reflejan que los razonamientos particulares son, a veces, más complejos que las tendencias. Como, por ejemplo, el razonamiento seleccionado para palabra clave LEY, quien utiliza la estrategia de división para descomponer su argumento: desde la perspectiva moral y la perspectiva legal. Para terminar el análisis de este grupo es también provechoso desplegar y comentar el flujo argumentativo:

Lo interesante de este flujo es que permite volver al argumento serial que se reconstruyó previamente con el material obtenido con el análisis de escalado multidimensional. A saber, que hay una tendencia de argumento circular, ya que las palabras clave deuda y justicia se refuerzan mutuamente en el razonamiento sin que se observe una causalidad necesaria: se está en deuda porque así lo determina la justicia, pagar la deuda es cuestión de justicia. Esta circularidad en el razonamiento probablemente se deba a un cruce entre el — supuesto - razonamiento técnico legal y el sentido común del hablante AM. Por su parte, la centralidad de la palabra clave cumplir, en relación directa con condena y ley, y cercana con correcto y condenado, demuestra que este grupo de AM se posiciona éticamente en sus argumentos a partir de un estricto sentido del deber. Vale decir, los AM de regiones, en su mayoría hombres, menores de 70 años, relativamente con un nivel de estudios más bajo, se orientarían por un cierto imperativo categórico. 


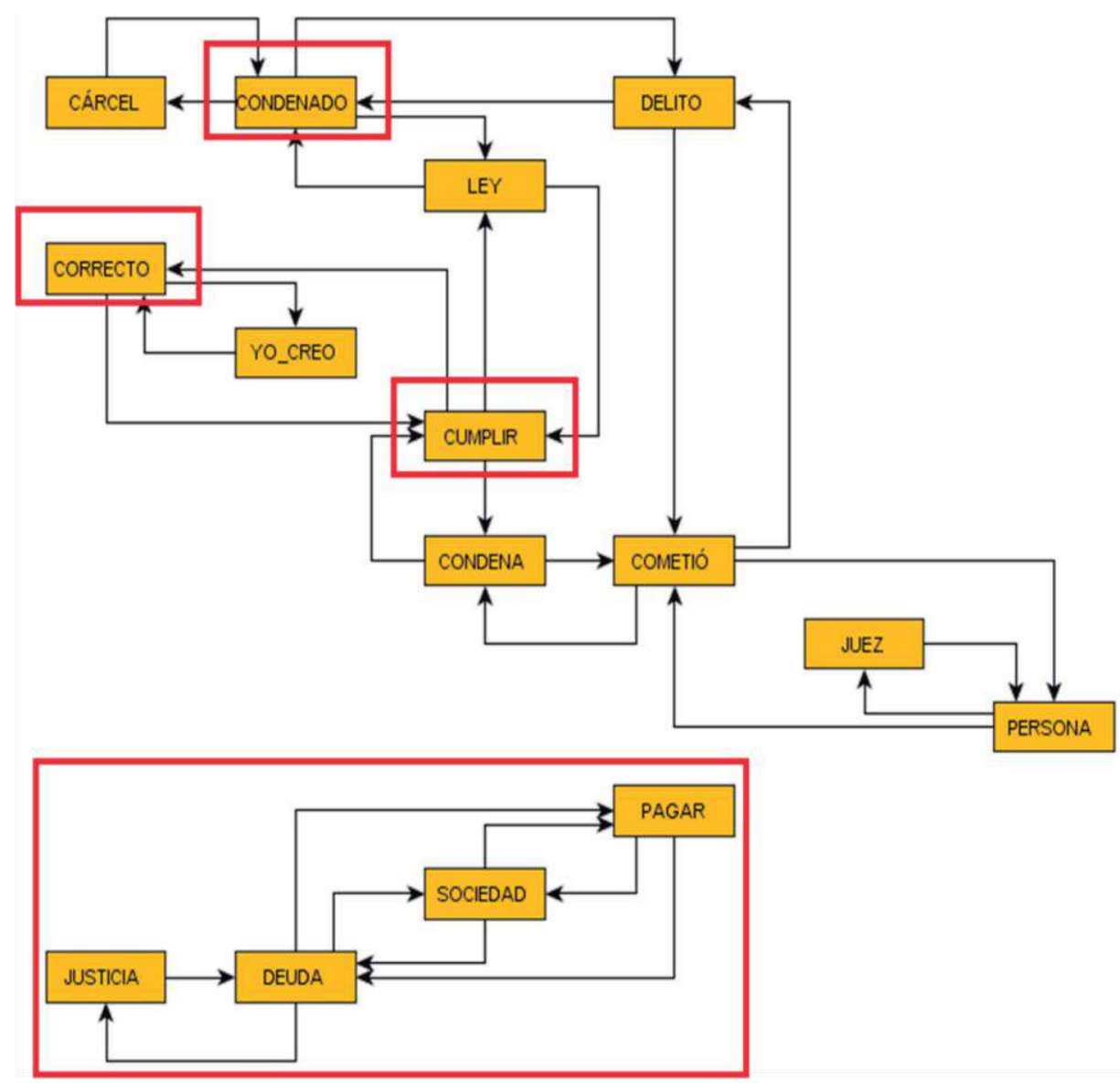

Figura 3. Estructura del flujo argumentativo de AM que apoyan que González denuncie

\subsubsection{AM que no saben si González debe denunciar a López}

En este segmento, los argumentos de los AM son similares entre sí, y muy consistentes en relación con los datos que utilizan:

Del despliegue visual del escalado multidimensional se desprende, principalmente, enunciados como los siguientes: Depende del delito que haya cometido la persona; yo creo que depende de la situación; con cierta distancia del punto de stress, 
también se puede configurar: ha pasado mucho tiempo; el hombre se ha transformado en una buena persona; López ya ha pagado su deuda con su actuar actual. En términos reconstructivos del argumento principal de la posición de este grupo, se da un argumento convergente (tres razones independientes apoyan una conclusión):

\section{Escalado multidimensional 2}

AM que no saben si González debe denunciar a López
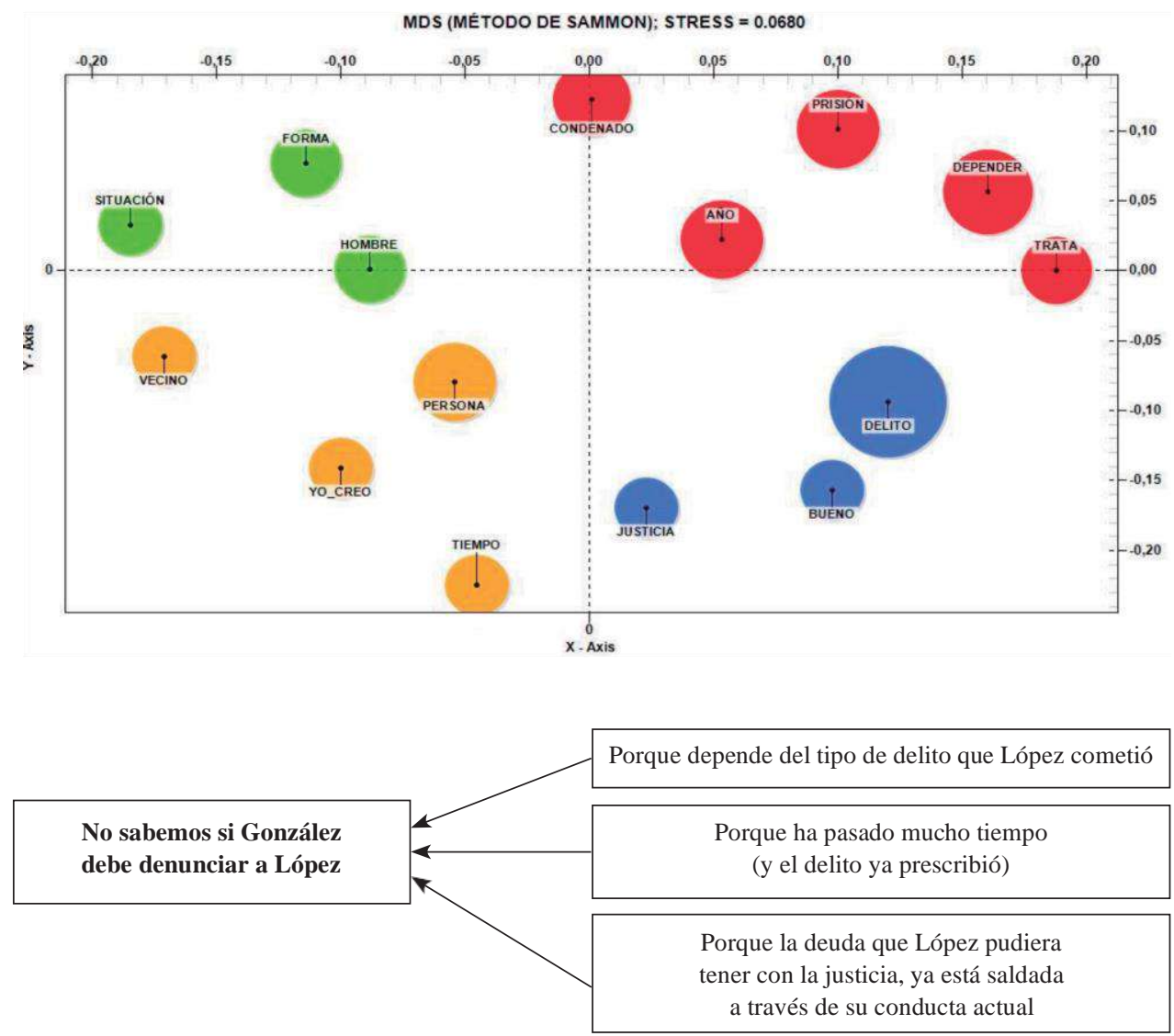

Figura 4. Argumento convergente

De AM que no saben si González debe denunciar a López

Es muy interesante observar que si el contexto de la investigación hubiese sido un grupo de discusión, este grupo, que es minoritario en las tendencias generales de la muestra, quizás se hubiese opuesto, interactiva y dialécticamente hablando, al grupo 
de quienes aprueban que González denuncie, ya que claramente los datos ponen en cuestión el punto de partida del grupo que aprueba denunciar. La perspectiva asumida, no obstante, es de cautela, dejando en evidencia que los AM que dudan (que recuérdese son mayormente de la capital del país, mujeres, por encima de los 70 años y con un nivel de estudios más alto) optan por lo que los epistemólogos (Feldman, 2005, 2007) llaman suspensión del juicio frente a la falta de información. Nótese que el concepto suspensión del juicio bien puede ser una manifestación de lo que Sperber et al. (2010) llaman, y que se indicó en la sección 3, vigilancia epistémica, vale decir ejercer una crítica más aguda cuando faltan elementos para adoptar un punto de vista definitivo.

Las asociaciones semánticas y dichos ejemplares de los AM de este grupo, confirman lo mencionado:
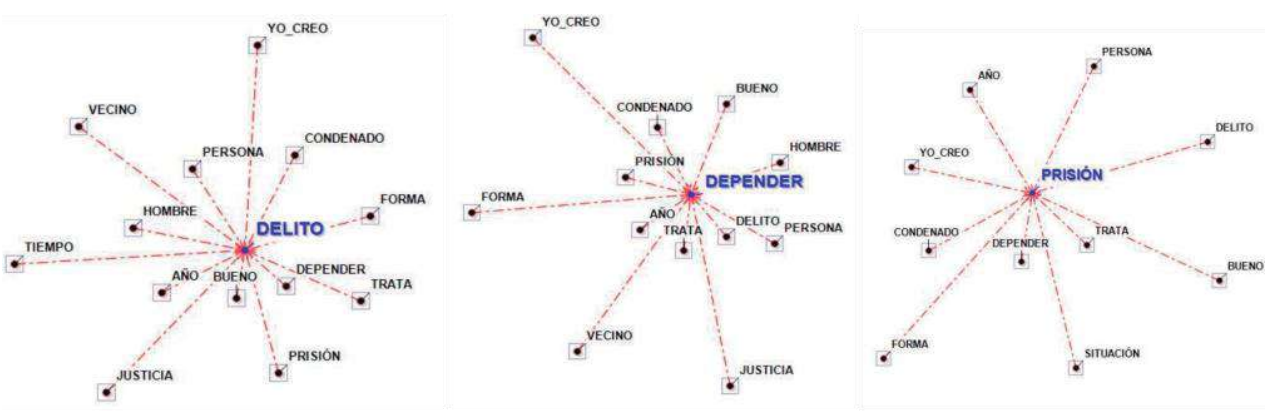

***** *IDnumber_000046*REGION_SANTIAGO *EDUCACION_MASEDUCA *EDAD_MASDE70 *SEXO_HOMBRE *RESPONDE NOSABE

Depende del delito por el cual fue condenado. Si se trata de abuso de menores, violación o similares, debe ser denunciado, pues constituye un peligro. Si se trata de condena por estafa, afiliación indebida, etc., no debe denunciar pues por su conducta se puede inferir que ha cambiado su forma de actuar y se ha reinsertado de buena forma a la sociedad - si va a prisión, no se obtiene nada bueno para la comunidad.

\begin{abstract}
**** *IDnumber 000087 *REGION CONCEPCION *EDUCACION MASEDUCA *EDAD MASDE70 *SEXO MUJER *RESPONDE NOSABE

Dificil respuesta. El denunciar al señor López dependería, para mí, de la causa por la que estaba condenado a 10 años de prisión, sobre todo si estamos hablando de chile hoy, con un sistema judicial muy objetable. Si se tratara de una condena por femicidio, homicidio, violación, o pedofilia, lo denunciaria sin dudarlo, previa conversación con el
\end{abstract}

\title{
***** *IDnumber_000121 *REGION_SERENA *EDUCACION_MENOSEDUCA *EDAD_HASTA70 *SEXO_MUJER
} *RESPONDE NOSABE

Yo creo que el señor González debería averiguar primero porque lo tenían preso, dependiendo de lo que haya hecho él podria llegar a tomar una decisión si dejarlo libre o ya que el cambió de visa y quiso ayudar a las demás personas. Y tratar de juzgar la sentencia que le fue impuesta en la prisión. Primero investigar todo y después formar una decisión.

\section{Figura 5. Asociaciones semánticas específicas y dichos ejemplares de AM que no saben si González debe denunciar a López}


Las asociaciones demuestran que la palabra clave PRISIÓN está cercanamente relacionada con DEPENDER, y que DEPENDER a su vez está ligada a AÑO, DELITO, TRATAR, PERSONAS. Esta red de vínculos es ilustrativa de una posición que pone en la balanza del juicio varios ángulos e información. Incluso, como se observa de la cita de una mujer AM de la ciudad de Concepción, comienza su reflexión señalando: "Difícil respuesta... Si se tratara de una condena por femicidio...". Esta estructura condicional es típica de escenarios contrafactuales, los que a su vez permiten observar razonamiento más complejo.

El flujo argumentativo aclara más aun esta red de vínculos, véase:

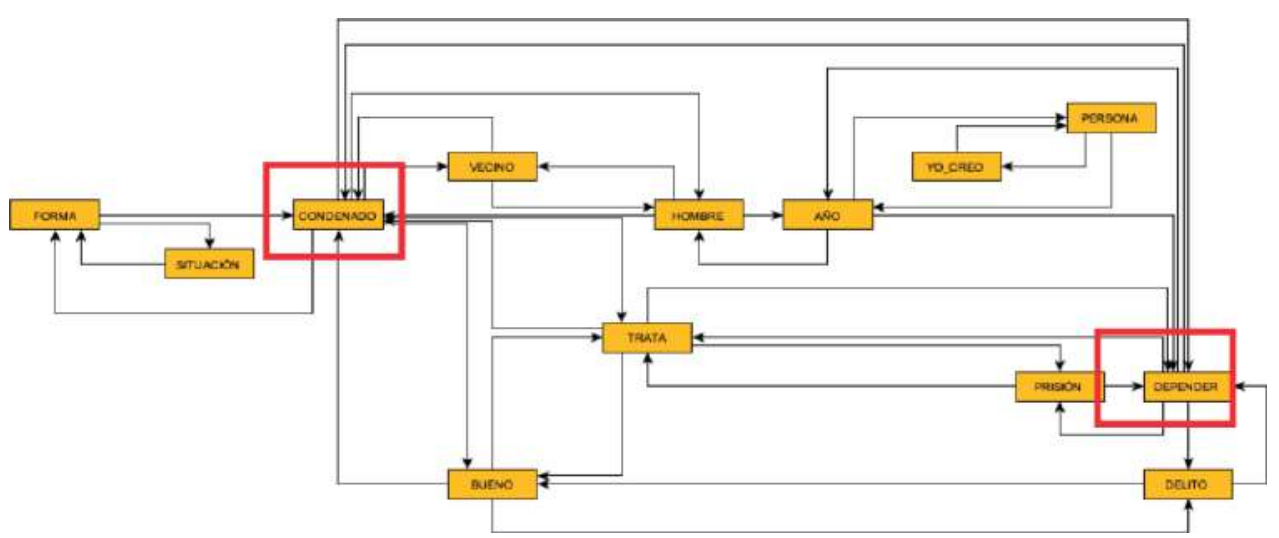

Figura 6. Estructura del flujo argumentativo de AM que no saben si González debe denunciar a López

La palabra clave CONDENADO tiene un flujo de vínculo denso, ligado a tratar bien a personas y ser un buen vecino, lo que viene a poner de relieve que esa condena se relativiza, ya que la red luego nos envía a la palabra clave DEPENDER, que funciona como eje principal, esto es, CONDENADO y DEUDA CON LA JUSTICIA Y LA SOCIEDAD dependen de cómo se consideren los hechos de BUEN VECINO y BUEN HOMBRE ACTUAL.

5.2.3. AM que rechazan que González denuncie a López

Así, llegamos al grupo de mayor concentración de AM: aquellos que se oponen a que González denuncie a López. Dada la cantidad de personas en este grupo, los 
análisis que se pueden realizar son más detallados y ricos en información. Por lo pronto, cabe destacar que respecto del grupo anterior, aquellos que dudan respecto de qué posición tomar, este grupo claramente mayoritario rechaza la posibilidad de denuncia por parte de González, precisamente por los datos, entre varios otros, de buen vecino y buen hombre actual, ya que los consideran hechos suficientes para respaldar adecuadamente sus argumentos a favor del rechazo.

Por ejemplo, se puede distinguir de entrada las dimensiones que estructuran los argumentos de los AM de este grupo, desarrollando un análisis factorial de correspondencias para resumir en pares conceptuales la manera en que se organiza el discurso contrario a denunciar a López. El análisis de esto arroja 4 ejes de posicionamiento argumentativo en virtud de dos factores, a saber: Factor 1, representado por el tópico UNA NUEVA PERSONA, con sus ejes Se regeneró y Cambió su vida; Factor 2, representado por el tópico UN BUEN CIUDADANO, con sus ejes Es un aporte a la sociedad y Ha pagado su crimen.

Ambos ejes, obviamente, apoyan decididamente que González no debe denunciar a López. Estos ejes quedan manifiestos con total claridad en el siguiente mapa factorial:

Los cuadrantes y colores de este mapa factorial permiten observar el tipo de palabras asociadas en cada orientación o énfasis de los ejes. Así, por ejemplo, tenemos que en la dirección de cambió su vida, en cuadrante de color rojo, palabras como recapacitar, cumplir, delito, se encuentran muy cercana a la posición de stress de mayor fuerza. Sucesivamente, al ver las palabras clave cercanas a ese punto de stress según eje y énfasis (y visualmente representadas por los distintos colores), la conceptualización general que apoya el argumento de rechazar la denuncia emerge con claridad. Este mapa factorial se ve reforzado con el escalado multidimensional que aporta una nueva visualización de palabras clave.

Tanto del mapa factorial como del escalado multidimensional, los enunciados que expresan argumentos en pro de que González no denuncie a López, se pueden reconstruir de la siguiente manera: cambió y ahora es útil a la sociedad; ha pagado su crimen con obras de caridad; se ha regenerado y merece una oportunidad; tiene una nueva vida y ayuda al prójimo; cambió su manera de actuar; yo creo que ahora es una persona buena. Estos enunciados reconstruidos con la información del mapa factorial y del escalado multidimensional, en términos de configuración argumentativa permiten graficar la posición como sigue: 


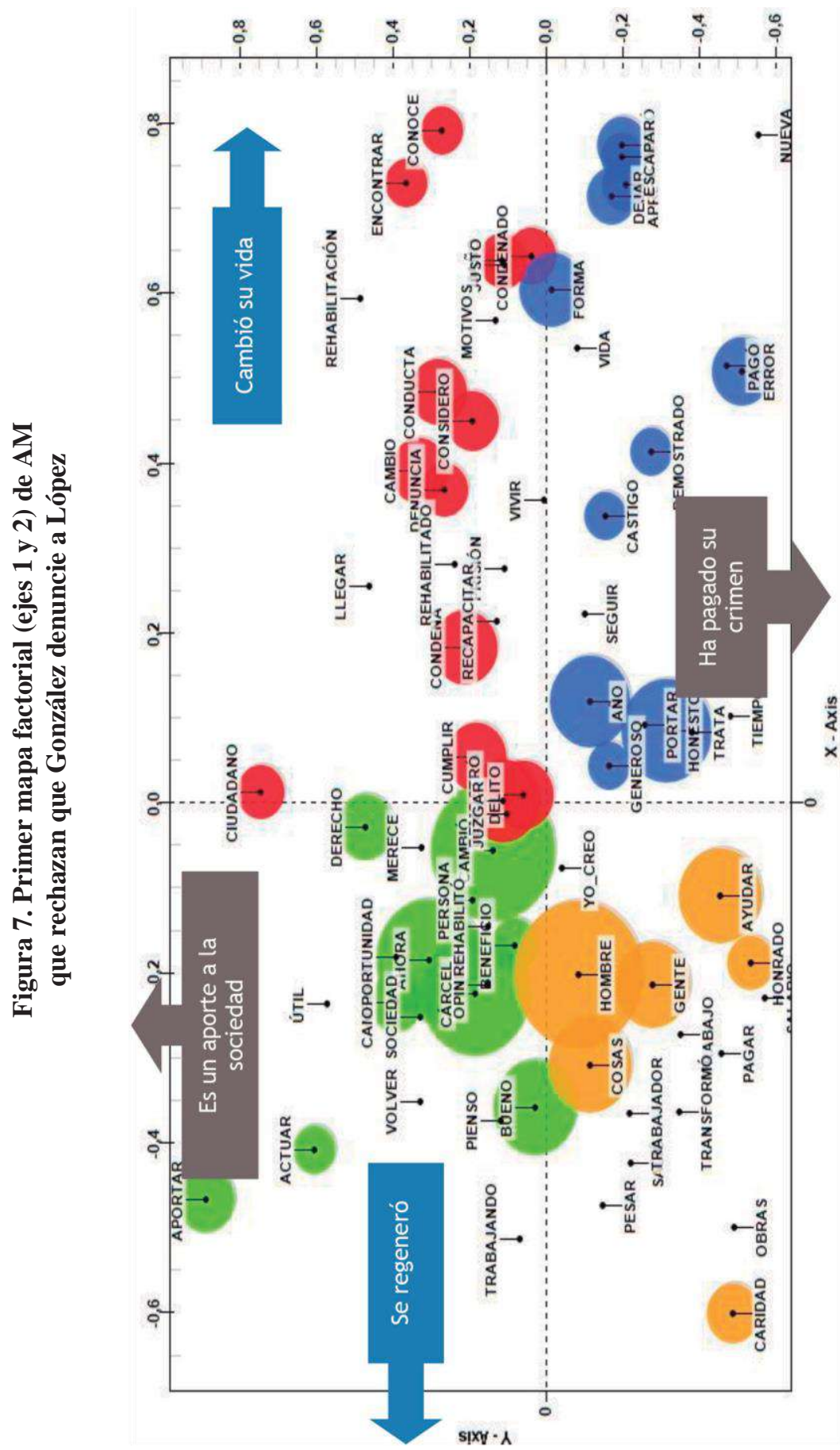

LINGÜÍSTICA Y LITERATURA 
Escalado multidimensional 3

AM que rechazan que González denuncie a López

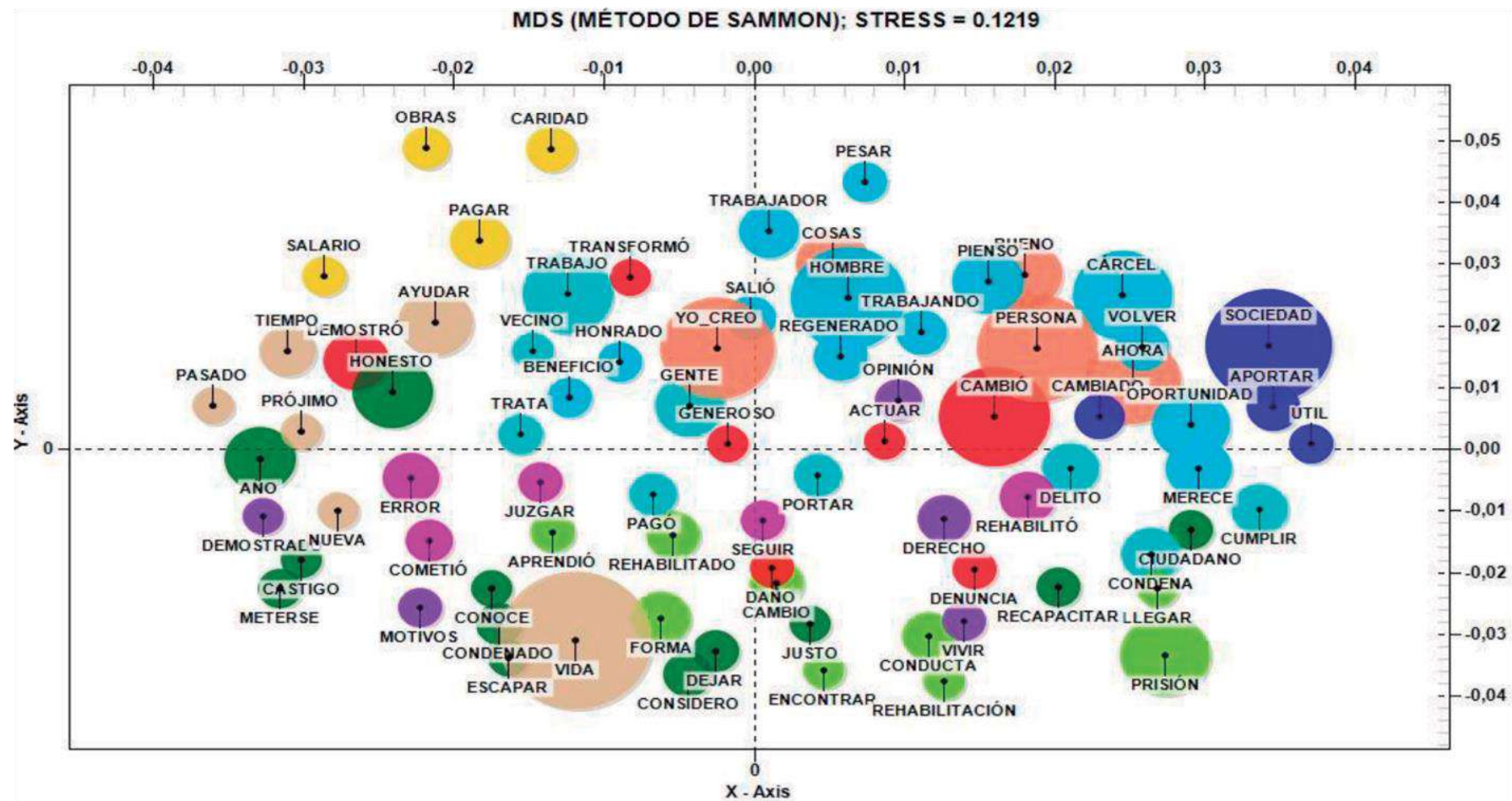




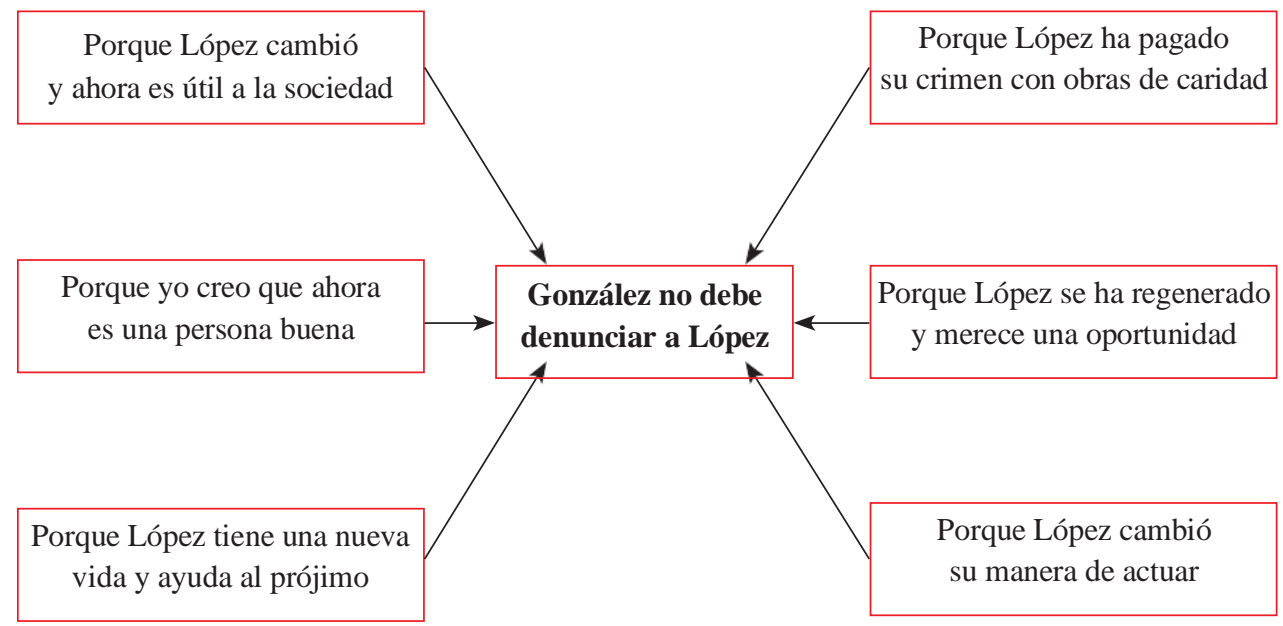

Figura 8. Argumento convergente

De AM que rechazan que González denuncie a López

El argumento convergente de este grupo es, como se observa claramente, más denso en términos de la cantidad de razones, del tipo de palabras clave utilizadas en cada razón, en la pertinencia de cada razón respecto del punto de vista, y también en la aceptabilidad de estas. Por supuesto, hay ciertas repeticiones en las razones, pero en ellas se observan campos argumentativos diversos. Las asociaciones semánticas específicas por palabras clave predominantes apoyan lo comentado hasta aquí. Véanse las siguientes asociaciones específicas:

Como bien se observa, las palabras clave y sus vínculos más cercanos, como por ejemplo en SOCIEDAD con AHORA, ÚTIL y APORTAR en cercanía inmediata, reflejan claramente la línea argumental de este grupo que reflexiona en torno al valor del cambio de actitud y/o al merecimiento de una nueva oportunidad. Se genera así la imagen de que este grupo de AM, por razones de empatía y generosidad y pragmatismo y/o utilidad, mayoritariamente conciben que no hay necesidad de mayor castigo a López. Se volverá sobre estas ideas en la sección de discusión y conclusiones. El flujo argumentativo, por su parte, también permite apreciar estos énfasis:

Aportando a las asociaciones, este flujo permite apreciar el rol que cumple la palabra clave DERECHO. La idea fuerza podría parafrasearse como sigue: las personas que han cambiado para aportar a la sociedad tienen el derecho a una nueva oportunidad de reinserción. 


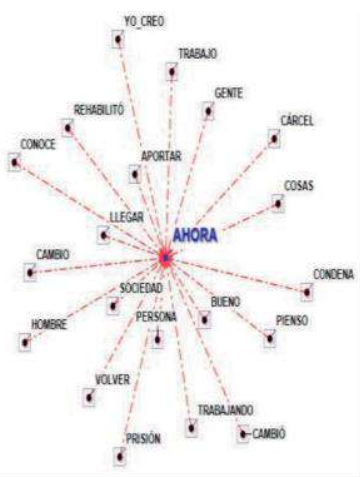

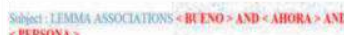
CHERSONA >

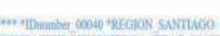

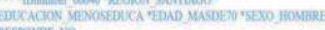

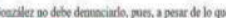

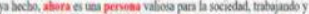

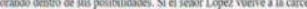

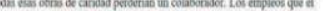

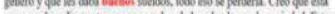
min opianic

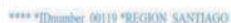

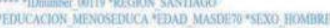
MesPONDE ND

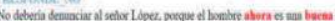
penobu y be menece ir a la ciacel

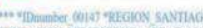

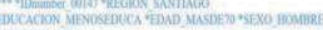

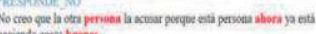

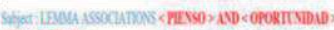
AD C YTRET >

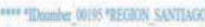

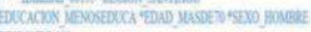
wesponac no

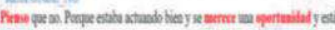

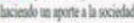

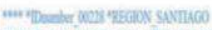

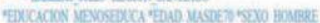
FESTONE Ni

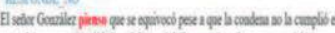

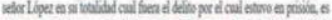

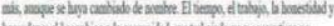

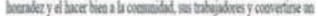

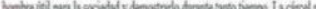

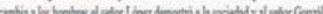

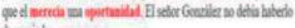
dencine.

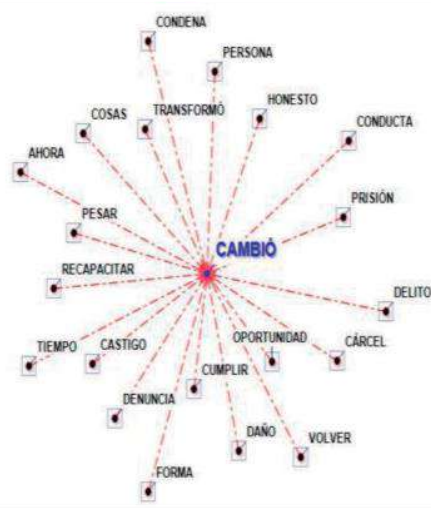

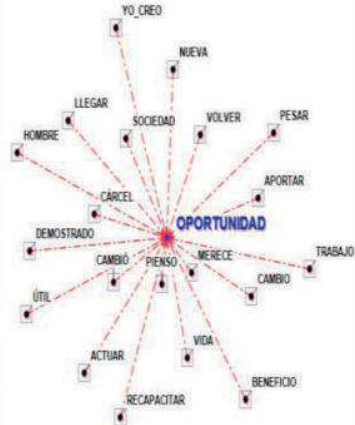

Sabject: LEMMA ASSOCAATONS \& CAMBIO > AND < HONESTO >

\section{w*t *Daumber 0000s5 *REGioN SANTUGC}

"EDUCACION MENOSEDUCA "EDAD MASDE70 -SEXO HOMBRE "RESPONDDE NO

Gouzalez no deberia denunciar a López ya que este cambio su estilo de vida, era housesto, tuvo su castigo. Gonzalez no deberia meterse ea los conflictos de López, cada uno sabe lo que hace, no debe ser intruso.

n..* *Damber 000155 "REGION SERENA

"EUUCACION MASEDUCA "EDAD HASTA70 *SEXO HOMBRE

No deberia hacerlo, pues el individuo cambio o se reformo, se transformó en un individuo honesto, afible y comprensivo.

*... थ Piumber 000215 *REGLON CONCEPCION

"EDUCACION MASFDUCA "EDAD HASTA70 "SEXO MUJEX TRESPONDE NO

Creo que no deberia ser denuaciado, solamente quedo un ato en prision, pero eso basto para cambiar su actitud, moato su negocio, era honesto. pagaba salanios altos a sus trabajaderes y ayudaba en obras de caricad, todas estas cosas demnestran que cambiaron sus valores eticos y morales y se denvesta ser el aejos demuesta ser d acjor medo de canbiar a taves del castigo camplir so sentencia solo le basto poco tiempo para poder cambiar y transformarse en

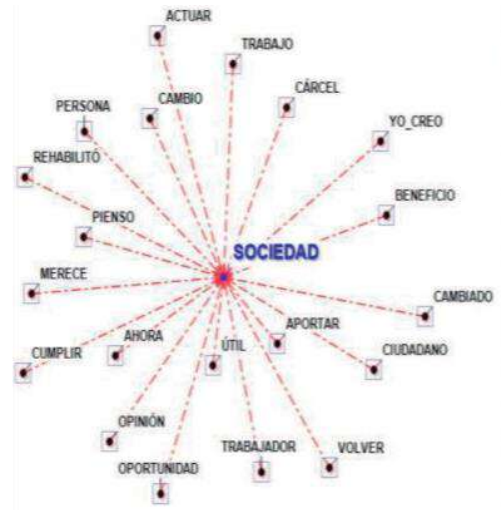

Subject : LEMMA ASSOCANIONS < SOCIFDAD > AVD < APORIAR

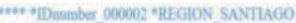

"EDUCACION MENOSFDUCA "EDAD MASDE70 *SEXO HOMAR: "RESPONDE NO

López no deberia ser denunciado porque abora esti obrando bien, ya cambiob y ahoca es un aporte a la sociedat.

*ur rIDumber 000012 *REGION SANTIACS

*EUCACION MIFNOSEDUCA *EAD MASDE70 *SEXO MUUER

THESPONDE NH

No porque se habia reincorporado a la weriedad, estaba aportando, ya habia cambiado, no hay que perjudicar a una persona que se rehabilito y que cumplia como ciudadano

*N. -1Diumber 000018 *RFGION SANTAGO

"EDUCACION MASEDUCA "EDAD MASDEZO *SEXO MUER "RESPONDE NO

No. porque el setor López se inserto y demortró ser ana persona ittil a si: mismo y la seciedad. Es uaa persona que aporta al grupo social en el cual

Figura 9. Asociaciones semánticas específicas y dichos ejemplares de AM que rechazan que González denuncie a López 


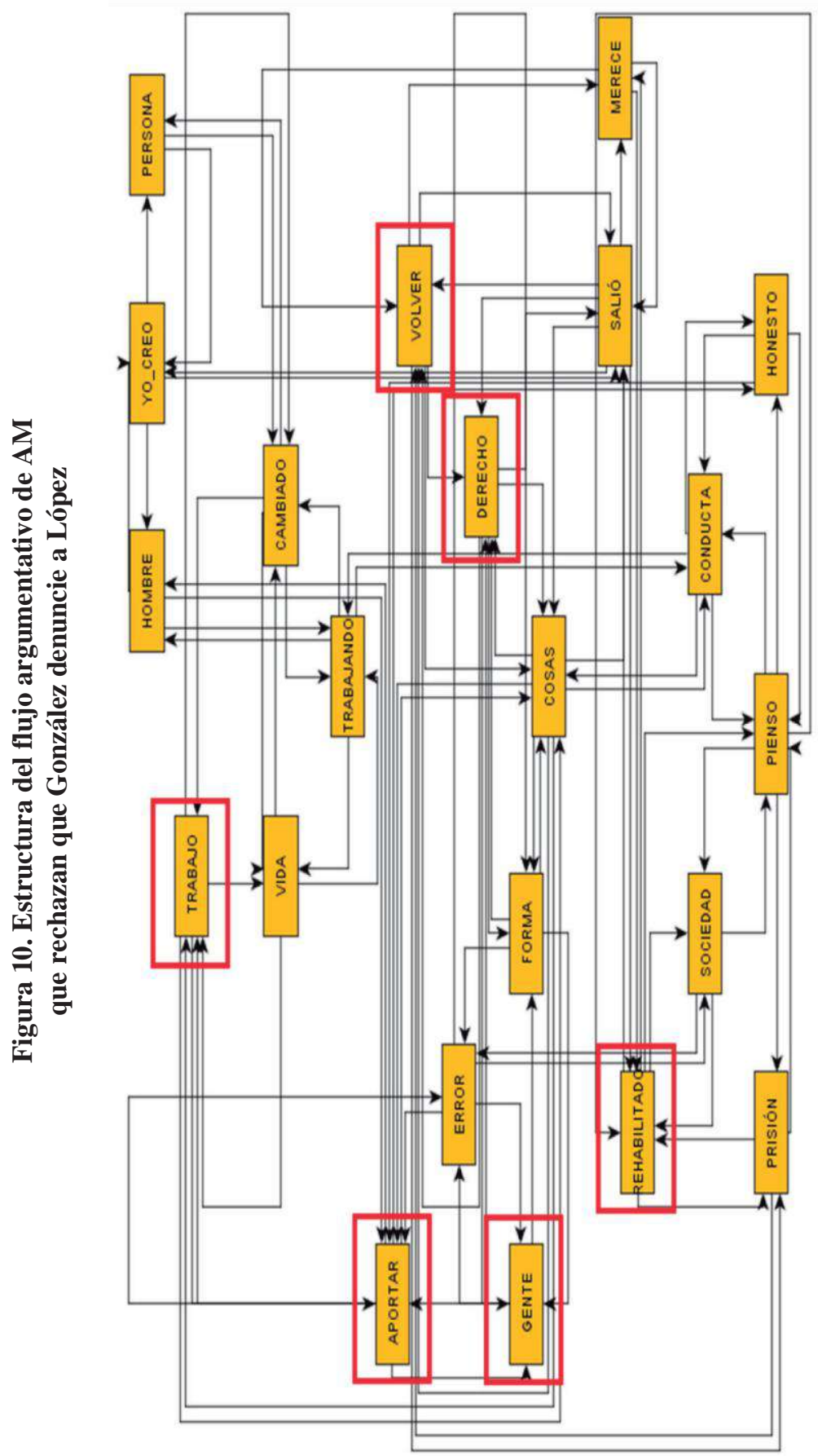


Figura 11. Palabras clave de cada agrupamiento en relación con ejes factoriales de AM que rechazan que González denuncie a López

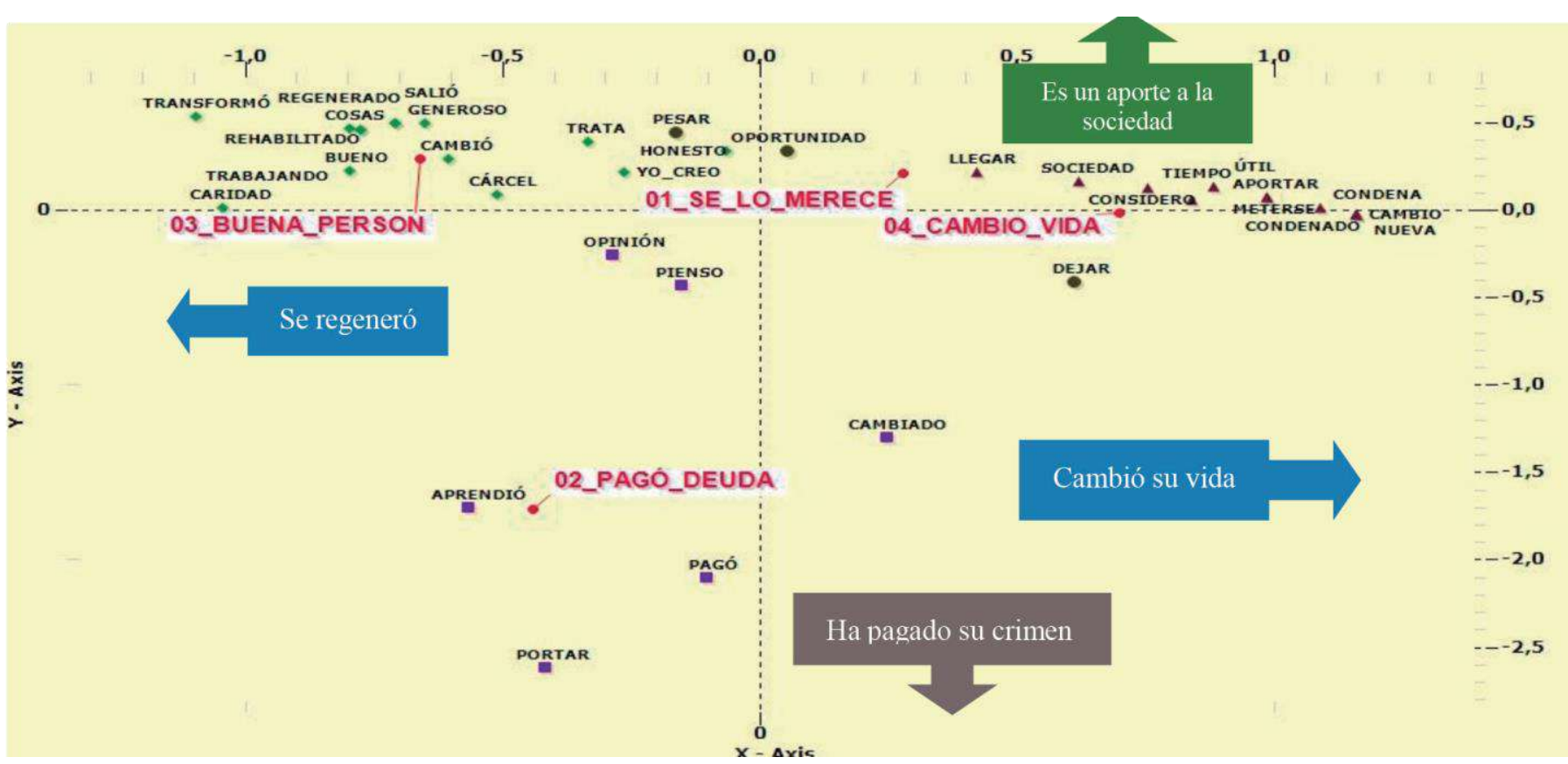




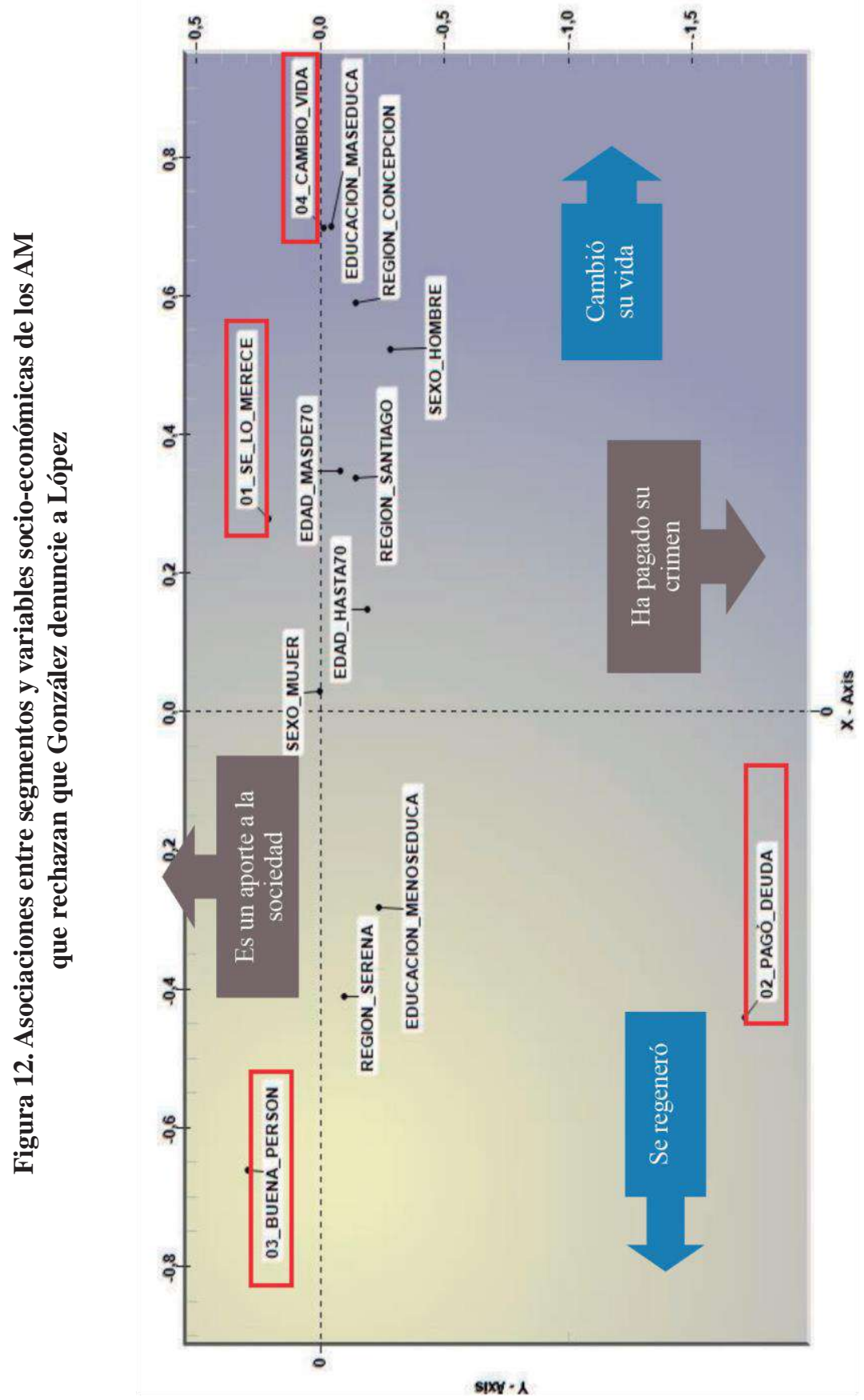


Como se apuntó al inicio de la sección 5.2., por el tamaño de este grupo los análisis que se pueden realizar son de mayor alcance. Por esta razón, a diferencia de los dos grupos anteriores, se complementará con un análisis de agrupamiento. Este análisis se obtiene a partir de un análisis de clasificación consistente en un método jerárquico ascendente, no supervisado, que opera en función de segmentaciones: la submuestra es dividida en segmentos y se caracteriza según el uso de determinados términos y/o enunciados claves que son más preponderantes en cada agrupamiento; además se realizará una asociación con las variables socio-económicas centrales del estudio: sexo, región y educación. Aplicado el procedimiento, un primer resultado es que de los enunciados clave en los AM que rechazan que González denuncie a López, un 14\% considera que López se merece una nueva oportunidad (agrupamiento 1), un $13 \%$ cree que López ya pagó su deuda con la sociedad (agrupamiento 2), un $42 \%$ considera que López es una buena persona (agrupamiento 3), y un $31 \%$ cree que López cambió su forma de vivir (agrupamiento 4).

La importancia de segmentar las tendencias y recobrar el enunciado que representa a cada agrupamiento radica en que al obtenerlo se accede a la razón principal que anima al punto de vista más robusto. Se considera predominantemente que López es una buena persona (agrupamiento 3), que es, entonces, la razón principal del punto de vista que rechaza la posibilidad de que González denuncie a López.

Con el objeto de descifrar qué palabras clave están asociadas a estos enunciados/ razones, y más aún, qué tipo de AM mayor está detrás de tales vínculos, véanse los dos siguientes mapas muy informativos:

Como siempre, la cercanía al punto de stress es determinante para la consideración de asociación entre una categoría, un segmento y palabras clave. Del último mapa, es importante enfatizar que las mujeres AM, sobre 70 años y más educadas, consideran que López, al haber cambiado su forma de actuar, merece una nueva oportunidad social, porque es una buena persona y un aporte al colectivo. Son este tipo de razones y entramados de razonamiento, en tanto hallazgos, los que a continuación se discutirán, utilizando conceptos provenientes de los estudios empíricos en torno a dilemas sociales y morales, a la vez que se indican algunos elementos centrales del tipo de agente argumentativo que subyace a cada grupo de AM que optó por una perspectiva sobre el contenido del dilema social.

\section{Discusión y conclusión}

Los razonamientos sobre el dilema social expuestos en los argumentos de los AM muestran al menos dos hechos. En primer lugar, la densidad argumentativa (en términos de cantidad y pertinencia de razones esgrimidas) de los AM que rechazan 
que se denuncie a López es claramente más robusta. Esto podría estar relacionado con que la información contenida en el propio dilema (relativa a las bondades del comportamiento de López posterior a su paso por la cárcel), provee más hechos y, por lo tanto, es más seguro, epistémicamente hablando, justificar un punto de vista. Por el contrario, aquellos que optan por que López sea denunciado, solo tienen como dato que se arrancó de la cárcel, sin saberse qué tipo de delito cometió. Esto último, precisamente, es lo que se esgrime como razón por el grupo minoritario que no sabe si denunciarlo o no: a falta de información precisa respecto al tipo de delito que López cometió, es difícil decantarse por un punto de vista, epistémicamente, bien defendido. Aquellos que optan por denunciar a López, utilizan como dato valores morales abstractos respecto de los que no están dispuestos a flexibilizar.

Para potenciar la discusión sobre estos hallazgos, se hace necesario utilizar algunas ideas provenientes de la literatura propia de los dilemas morales y sociales. En la literatura contemporánea (van Lange, Rockenbach \& Yamagishi, 2014), la discusión ha girado, entre otros de sus puntos centrales, en torno a las disposiciones que tenemos los humanos a apoyar (o premiar) y castigar a las personas que cooperan o no cooperan. De acuerdo con los resultados en este ámbito, la predisposición a cooperar con los cooperadores es más fuerte que la predisposición a castigar a los que no cooperan y/o engañan. Es decir, la literatura señala que podemos sancionar castigando de manera ejemplar (agresivamente), pero solo lo hacemos de esta forma cuando no hay alternativas. La energía estaría puesta en sancionar premiando (cultivar, potencia, motivar la conducta cooperadora), en vez de sancionar negativamente, cuando existe alternativa. Este sería un diseño evolutivo mucho más potente para cuidar la existencia del grupo (motivar conducta cooperadora) que estar preocupados constantemente (como una suerte de estado policial) de buscar a las personas que están engañando y no cooperando. Desde este punto de vista, tiene mucho sentido el resultado que se ha obtenido en esta investigación. La tendencia mayoritaria de sancionar positivamente a López a través de no denunciarlo estaría en relación con el reconocimiento que hacen los AM de los esfuerzos de López por cambiar su comportamiento antisocial a uno empático, generoso y beneficioso.

Es muy interesante notar que la manifestación argumentativa de los AM que estiman que López no debe ser denunciado es mucho más diversificada que la del grupo que estima denunciar y aquellos que no saben si hacerlo. Se podría decir: en una situación problemática o controversial, la tendencia natural a premiar a los cooperadores está en relación directamente proporcional con la densidad argumentativa. Esta diversidad se observa tanto en las asociaciones semánticas como en el flujo argumentativo. Por ejemplo, en ellos hay mayor sinonimia en la red semántica y las palabras clave se asocian con mayor cercanía con términos relevantes del intertexto. 
Por su parte, el grupo de AM que dudan de si denunciar a López, demuestra un flujo argumentativo que está, precisamente, en una gradación media respecto de los que no denuncian y de los que sí lo harían, confirmando de alguna forma que a mayor empatía, o tendencia utilitarista, mayor riqueza argumentativa. Los AM que denunciarían a López quedan anclados sólo en el hecho de que López arrancó de la cárcel y eliminan los datos de cooperación que la conducta posterior de López muestra. Como se sabe (van Eemeren \& Grootendorst, 2004), una dimensión teórica y empírica fundamental de la argumentación es la razonabilidad con la que un sujeto manifiesta su competencia. La razonabilidad de un hablante se observa cuando éste es capaz de resolver conflictos de opinión de manera crítica. Investigar en qué circunstancias se encuentra esta dimensión crucial de la actividad argumentativa en AM ayudará particularmente en el esclarecimiento y definición de mecanismos de interacción controversial con este grupo etario, en una sociedad como la chilena que se encuentra en transformaciones esenciales de su carácter cultural. Y los resultados que se reportan aquí ayudan en esa dirección. Los AM que no denunciarían a López declaran con claridad su punto de vista y asumen también con mayor claridad el contexto controversial en el que se encuentran; asumen el rol dialéctico y defienden su punto de vista por medio del uso de mayor cantidad de razones; profundizan cualitativamente e incrementan cuantitativamente la oferta, ante el oponente, de razones y esquemas argumentativos para defender el punto de vista asociado al rol dialéctico asumido. Desde el punto de vista del lado opuesto, es decir, desde la perspectiva del que no premia al cooperador, el trabajo de la política pública y educativa versaría sobre ejercitarlos en la actividad crítica, que supone asumir una posición flexible cuando las acciones de los sujetos ya son beneficiosas para el grupo.

\section{Referencias bibliográficas}

1. Arancibia, B., Véliz, M., Riffo, B. \& Roa-Ureta, R. (2014). Procesamiento de cláusulas relativas anidadas, memoria operativa y envejecimiento. RLA, 52(1), pp. 155-179.

2. Burke, D. \& Shafto, M. (2008). Language and Aging. En F. Craik \& T. Salthouse (Eds.), The handbook of aging and cognition (pp. 373-443). Mahwah, NJ: Erlbaum Associates, Inc.

3. Caplan, D., Dede, G., Waters, G., Michaud, J. \& Tripodis, Y. (2011). Effects of age speed of processing and working memory on comprehension of sentences with relative clauses. Psychology and Aging, 26(2), pp. 439-450

4. Condret-Santi, V., Barbeau, E., Matharan, F., Le Goff, M., Dartigues, J. \& Amieva, H. (2015). Prevalence of Word Retrieval Complaint and Prediction of Dementia in a Population-Based Study of Elderly Subjects. Dementia \& Geriatric Cognitive Disorders, 35(5/6), pp. 313-324.

5. Crespo, N. (1995). El desarrollo ontogenético del argumento. Revista Signos, 18(37), pp. 69-82.

6. Dede, G., Caplan, D., Kemtes, K. \& Waters, G. (2004). The Relationship Between Age Verbal Working Memory, and Language comprehension. Psychology and Aging, 19(4), pp. 601-616. 
7. Ducrot, O. (2004). Sentido y argumentación. En E. Arnoux \& M. García (Eds.), Homenaje a Oswald Ducrot (pp. 359-370). Buenos Aires: Eudeba.

8. Ducrot, O. \& Carel, M. (2006). La semántica argumentativa. Una introducción a la teoría de los bloques semánticos. Buenos Aires: Colihue.

9. Eemeren, F. van. \& Grootendorst, R. (2004). A Systematic Theory of Argumentation. The PragmaDialectical Approach. Cambridge: Cambridge University Press.

10. Eemeren, F. van. (2010). Strategic Maneuvering in Argumentative Discourse. Extending the Pragma-Dialectical Theory of Argumentation. Amsterdam: John Benjamins.

11. Eemeren, F. van, Garssen, B. \& Meuffels, B. (2009). Fallacies and Judgments of Reasonableness. Empirical Research Concerning the Pragma-Dialectical Discussion Rules. New York: Springer.

12. Fabrigoule, C., Bareberger-Gateau, F. \& Dartigues, J. F. (2006). The PAQUID study. En H. A. Tuokko \& D. F. Hultsch (Eds.), Mild Cognitive Impairment. International Perspectives (pp. 3155). Nueva York: Taylor \& Francis.

13. Feldman, R. (2005). Deep Disagreement, Rational Resolutions and Critical Thinking. Informal Logic, 25 (1), pp. 13-23.

14. Feldman, R. (2007). Reasonable Religious Disagreement. En L. M. Antony, (Ed.), Philosophers Without Gods: Meditations on Atheism and the Secular Life (pp. 194-214). Oxford: Oxford University Press.

15. García, M. (por aparecer). El enfoque dialógico de la argumentación y la polifonía, puntos de vista evidenciales y puntos de vista alusivos. Revista de Filología Hispánica.

16. Gollan, T. \& Brown, A. (2006). From tip-of-the-tongue (TOT) Data to Theoretical Implications in Two Steps: When more TOTs Means Better Retrieval. Journal of Experimental Psychology, 135, pp. 462-483.

17. Hammer, L. \& Noemi, C. (2015). Relación entre pensamiento crítico y complejidad discursiva en estudiantes universitarios. Revista Onomazein, 32, pp. 184-197.

18. Hample, D. (2005). Arguing: Exchanging Reasons Face to Face. Mahwah, New Jersey: Lawrence Erlbaum Associates, Inc.

19. Hample, D., Warner, B. \& Young, D. (2009). Framing and Editing Interpersonal Arguments. Argumentation, 23, pp. 21-37

20. Hample, D. \& Anagondahalli, D. (2015). Understandings of Arguing in India and the United States: Argument Frames, Personalization of Conflict, Argumentativeness, and Verbal Aggressiveness. Journal of Intercultural Communication Research, 44, 1-26.

21. Hu, X. (2017). Must a Successful Argument Convert an Ideal Audience? Argumentation, 31 (1), pp. 165-177.

22. Instituto Nacional de Estadísticas (2017). http://www.censo2017.cl/wp-content/uploads/2017/12/ Presentacion_Resultados_Definitivos_Censo2017.pdf, recuperado el 9 de febrero de 2018.

23. Johnson, R. (2000). Manifest Rationality. A Pragmatic Theory of Argument. New Jersey: Lawrence Erlbaum Associates, Inc.

24. Johnson, R. \& Blair, A. (1977). Logical self-defense. Toronto: McGraw-Hill Ryerson.

25. Juncos, O., Pereiro, A. \& Rodríguez, M. (2005). Narrative speech in aging: Quantity, information content, and cohesion. Brain and Language, 95, pp. 423-434. 
26. Juncos, O., Facal, D., Álvarez, M. \& Rodríguez M.S. (2006). El fenómeno de la punta de la lengua (PDL) en el proceso de envejecimiento. Psicothema, 18(3)3, pp. 500-505.

27. Kemper, S. (1992). Language and aging. En F.I. Craik \& T. Salthouse (Eds.), The handbook of aging and cognition (pp. 213-270). Hillsdale N. J.: Erlbaum.

28. Kemper, S., Herman, R. \& Chiung-Ju, L. (2004). Sentence production by young and older adults in controlled contexts. Journal of Gerontology: Psychological Science, 59B, pp. 230-234.

29. Kemper, S., Herman, R. \& Lian, C. (2003). The Costs of Doing Two Things at Once for Young and Older Adults: Talking While Walking, Finger Tapping, and Ignoring Speech or Noise. Psychology and Aging, 18(2), pp. 181-192.

30. Kemper, S., Thompson, M. \& Marquis, J. (2001). Longitudinal change in language production: Effects of aging and dementia on grammatical complexity and propositional content. Psychology and Aging, 16, pp. 600-614.

31. Kemper, S. \& Sumner, A. (2001). The Structure of Verbal Abilities in Young an Older Adults. Psychology and Aging, 16, pp. 312-322.

32. Kemper, S., Greiner, L., Marquis, J., Prenovost, K. \& Mitzner, T. (2001). Language Decline Across the Life Span: Findings From the Nun Study. Psychology and Aging, 16, pp. 227-239.

33. Lange, P. van, Rockenbach, B. \& Yamagishi, T. (2014). Reward and Punishment in Social Dilemmas. New York: Oxford University Press.

34. Mackenzie, C. (2000). Adult Spoken Discourse: The Influences of Age and Education. International Journal of Language and Communication Disorders, 35, pp. 269-285.

35. Martín-Aragoneses, M. \& Fernández-Blázquez, M. (2012). El lenguaje en el envejecimiento: Procesos de recuperación léxica. Revista de Logopedia, Foniatría y Audiología, 32(2), pp. 34-46.

36. Mercier, H. (2011a). When Experts Argue: Explaining the Best and the Worst of Reasoning. Argumentation, 23(3), pp. 313-327.

37. Mercier, H. (2011b). Reasoning Serves Argumentation in Children. Cognitive Development, 26(3), pp. 177-191.

38. Mercier, H. (2016). The Argumentative Theory: Predictions and Empirical Evidence. Trends in Cognitive Sciences, 20(9), pp. 689-700.

39. Mercier, H. \& Sperber, D. (2017). The Enigma of Reason. A New Theory of Human Understanding. London: Allen Lane.

40. Migdalek, M., Santibáñez, C. \& Rosemberg, C. (2014). Estrategias argumentativas en niños pequeños: Un estudio a partir de las disputas durante el juego en contextos escolares. Revista Signos, 47(86), pp. 435-462.

41. Padilla, C. \& López, E. (2011). Grados de complejidad argumentativa en escritos de estudiantes universitarios de humanidad. Revista Praxis, 13(20), pp. 61-90.

42. Palmer, K., Bäckman, L., Small, B. \& Fratiglioni, L. (2006). Cognitive Impairment in Elderly Person Without Dementia: Findings From the Kungsholmen Project. En H. Tuokko \& D. Hultsch (Eds.), Mild Cognitive Impairment. International Perspectives (pp. 57-75). New York: Taylor \& Francis.

43. Parodi, G. (2000). La evaluación de la producción de textos escritos argumentativos: una alternancia cognitivo/ discursiva. Revista Signos, 33 (47), pp. 151-16. 
44. Peronard, M. (1991). Antecedentes ontogenéticos de la argumentación. En E. Luna (Coord.), Scripta philologica: In honorem Juan M. Lope Blanch (pp. 417-443). México: Universidad Autónoma de México.

45. Quiroga, P., Albala, C. \& Klaassen, G. (2004). Validación de un test de tamizaje para el diagnóstico de demencia asociada a edad, en Chile. Revista Médica de Chile, 132(4), pp. 467-478.

46. Ricco, R. \& Sierra, A. (2011). Individual Differences in the Interpretation of Commitment in Argumentation. Argumentation, 25, pp. 37-61.

47. Riffo, B. \& Benoit, C. (2007). Procesamiento de oraciones con incrustación central y derecha en adultos jóvenes y AM. Revista de Lingüística Teórica y Aplicada, 45 (1), pp. 13-31.

48. Santibáñez, C. (2014). ¿Para qué sirve argumentar? Problematizando teórica y empíricamente el valor y la función de la argumentación. Revista Círculo de Lingüística Aplicada a la Comunicación, 58, pp. 163-205.

49. Santibáñez, C. \& Hample, D. (2015). Orientations Toward Interpersonal Arguing in Chile. Pragmatics, 23 (3), pp. 453-476.

50. Santibáñez, C., Migdalek, M. \& Rosemberg, C. (2016). Estilos argumentativos en jóvenes universitarios chilenos: Implicancias para una pedagogía colectiva. En Santibáñez, C. (Comp.), Ecología argumentativa universitaria: desde la realidad a los conceptos (pp. 161-186). Concepción: Cosmigonon.

51. Schär, R. (2011). Uses of Arguments from Definition in Children's Argumentation. En P. Bondy \& L. Benacquista (Eds.), Argumentation, Objectivity, and Bias: Proceedings of the $11^{\text {th }}$ International Conference of the Ontario Society for the Study of Argumentation (pp. 1-15), 18-21 May 2016. Windsor, ON: OSSA.

52. Schär, R. (2017). Definitional Arguments in Children's Speech. L'analisi Linguistica e Letteraria, 1, 173-192.

53. Sperber, D. \& Mercier, H. (2012). Reasoning as a Social Competence. En H. Landemore \& J. Elster (Eds.), Collective Wisdom: Principles and Mechanisms (pp. 368-392). Cambridge: Cambridge University Press.

54. Singer, T., Verhaeghen, P., Ghisletta, P., Lindenberger, U. \& Baltes, P. B. (2003). The Fate of Cognition in Very Old Age: Six Years Longitudinal Findings in the Berlin Aging Study (BASE). Psyhology and Aging, 18, pp. 318-331.

55. Tapia-Ladino, M. (2005). Producción de habla y fenómenos de vacilación en la conversación de adultos jóvenes y AM. Anales de Psiquiatria, 21(5), pp. 215-222.

56. Tindale, C. (2004). Rhetorical Argumentation. New York: Sage.

57. Tomasello, M. (2014). A Natural History of Human Thinking. Cambridge: Harvard University Press.

58. Toulmin, S., Rieke, R. \& Janik, A. (1979). An Introduction to Reasoning. New York: Macmillan.

59. Ulatowska, H. (Ed). (1985). The Aging Brain. Communication in the Elderly. Boston: CollegeHill.

60. Véliz, M. (2004). Procesamiento de estructuras sintácticas complejas en AM y adultos jóvenes. Estudios Filológicos, 39, pp. 65-81.

61. Véliz, M., Riffo, B., Aguilar, V. \& Sáez, K. (2011). Procesamiento de oraciones ambiguas de vía muerta y envejecimiento: un estudio experimental. Revista Onomazein, 24(2), pp.199-222. 
62. Véliz, M., Riffo, B., Hernández, M., Sáez, Y.\& Sáez, K. (2013). Oraciones producidas por AM y adultos jóvenes en una situación controlada. Revista Onomazein, 27, pp. 241-257.

63. Véliz, M., Riffo, B. \& Vásquez, A. (2009). Recuerdo inmediato de oraciones de sintaxis compleja en adultos jóvenes y mayores. Estudios Filológicos, 44, pp. 243-258.

64. Walton, D. (2007). Media Argumentation. Dialectic, Persuasion, and Rhetoric. Cambridge: Cambridge University Press.

65. Walton, D. (2013). Methods of Argumentation. New York: Cambridge University Press.

66. Wilson, R., Beckett, L., Barnes, L., Schneider, J., Bach, J., Evans, D. \& cols. (2002). Individual Difference in Rates of Change in Cognitive Abilities of Older Persons. Psychology and Aging, 17, pp. 179-193.

67. Wingfield, A. \& Stine-Morrow, E. (2000). Language and Speech. En F. Craik \& T. Salthouse (Eds.), The Handbook of Aging and Cognition (pp. 359-416). Mahwah, NJ: Lawrence Erlbaum Associates, Inc.

68. Xie, Y., Hample, D. \& Wang, X. (2015). A Cross-Cultural Analysis of Argument Predispositions in China: Argumentativeness, Verbal Aggressiveness, Argument Frames, and Personalization of Conflict. Argumentation, 29, pp. 265-284. 